\title{
COMMUTING INVOLUTIONS AND DEGENERATIONS OF ISOTROPY REPRESENTATIONS
}

\author{
DMITRI I. PANYUSHEV
}

\begin{abstract}
Let $\sigma_{1}$ and $\sigma_{2}$ be commuting involutions of a semisimple algebraic group $G$. This yields a $\mathbb{Z}_{2} \times \mathbb{Z}_{2}$-grading of $\mathfrak{g}=\operatorname{Lie}(G), \mathfrak{g}=\bigoplus_{i, j=0,1} \mathfrak{g}_{i j}$, and we study invarianttheoretic aspects of this decomposition. Let $\mathfrak{g}\left\langle\sigma_{1}\right\rangle$ be the $\mathbb{Z}_{2}$-contraction of $\mathfrak{g}$ determined by $\sigma_{1}$. Then both $\sigma_{2}$ and $\sigma_{3}:=\sigma_{1} \sigma_{2}$ remain involutions of the non-reductive Lie algebra $\mathfrak{g}\left\langle\sigma_{1}\right\rangle$. The isotropy representations related to $\left(\mathfrak{g}\left\langle\sigma_{1}\right\rangle, \sigma_{2}\right)$ and $\left(\mathfrak{g}\left\langle\sigma_{1}\right\rangle, \sigma_{3}\right)$ are degenerations of the isotropy representations related to $\left(\mathfrak{g}, \sigma_{2}\right)$ and $\left(\mathfrak{g}, \sigma_{3}\right)$, respectively. We show that these degenerated isotropy representations retain many good properties. For instance, they always have a generic stabiliser and their algebras of invariants are often polynomial. We also develop some theory on Cartan subspaces for various $\mathbb{Z}_{2}$-gradings associated with the $\mathbb{Z}_{2} \times \mathbb{Z}_{2}$-grading of $\mathfrak{g}$.
\end{abstract}

\section{CONTENTS}

Introduction 1

1. Generalities on involutions and isotropy representations 4

2. Quaternionic decompositions, triads, and dyads 6

3. Comparing Cartan subspaces 9

4. Degenerations of symmetric spaces and isotropy representations 14

5. Invariants of certain degenerated isotropy representations 21

$\begin{array}{ll}\text { 6. Problems and observations } & 27\end{array}$

$\begin{array}{ll}\text { References } & 30\end{array}$

\section{INTRODUCTION}

The ground field $\mathbb{F}$ is algebraically closed and char $F=0$. Let $\sigma_{1}$ and $\sigma_{2}$ be commuting involutions of a connected semisimple algebraic group $G$. This yields a $\mathbb{Z}_{2} \times \mathbb{Z}_{2}$-grading of $\mathfrak{g}=\operatorname{Lie}(G)$ :

$$
\mathfrak{g}=\bigoplus_{i, j=0,1} \mathfrak{g}_{i j}, \text { where } \mathfrak{g}_{i j}=\left\{x \in \mathfrak{g} \mid \sigma_{1}(x)=(-1)^{i} x \quad \& \quad \sigma_{2}(x)=(-1)^{j} x\right\} .
$$

2010 Mathematics Subject Classification. 13A50, 14L30, 17B40, 22 E46.

Key words and phrases. Semisimple Lie algebra, $\mathbb{Z}_{2}$-contraction, Cartan subspace, quaternionic decomposition, algebra of invariants. 
Since $\sigma_{1}, \sigma_{2}$, and $\sigma_{3}=\sigma_{1} \sigma_{2}$ are pairwise commuting involutions, this decomposition possesses an $\mathbb{S}_{3}$-symmetry, and following [24] we say that $(0 \cdot 1)$ is a quaternionic decomposition. For, if $(\alpha, \beta, \gamma)$ is any permutation of the indices $(01,10,11)$, then $\left[\mathfrak{g}_{00}, \mathfrak{g}_{\alpha}\right] \subset \mathfrak{g}_{\alpha}$ and $\left[\mathfrak{g}_{\alpha}, \mathfrak{g}_{\beta}\right] \subset \mathfrak{g}_{\gamma}$. Various problems related to this structure (in the setting of Lie groups or Lie algebras) has been studied before. Let $G^{\sigma_{i}}$ be the fixed-point subgroup of $\sigma_{i}$. In [13], Matsuki obtained fundamental results on $\left(G^{\sigma_{1}}, G^{\sigma_{2}}\right)$ double cosets in $G$. The quotient variety $G / /\left(G^{\sigma_{1}} \times G^{\sigma_{2}}\right)$ has been studied by Helminck and Schwarz [7, 8]. In [24], Vergne applies quaternionic decompositions to the study of the Kostant-Sekiguchi correspondence. The conjugacy classes of pairs of commuting involutions are classified, see [9] and references therein. In [25], commuting conjugate involutions $\sigma_{1}, \sigma_{2}, \sigma_{3}$ (triads) are used in the proof of Rosenfeld's conjecture on the existence of elliptic planes over the tensor product of two composition algebras.

In this article, we consider some other aspects of quaternionic decomposition $(0 \cdot 1)$. This decomposition embraces several $\mathbb{Z}_{2}$-gradings of $\mathfrak{g}$ and the subalgebras $\mathfrak{g}^{\sigma_{i}}=\operatorname{Lie}\left(G^{\sigma_{i}}\right)$. The involutions $\sigma_{1}, \sigma_{2}, \sigma_{3}$ are said to be big and the induced involutions of $\mathfrak{g}^{\sigma_{1}}, \mathfrak{g}^{\sigma_{2}}, \mathfrak{g}^{\sigma_{3}}$ are said to be little. The same terminology applies to the corresponding $\mathbb{Z}_{2}$-gradings, symmetric spaces, and Cartan subspaces (= CSS for short). Our ultimate goal is to elaborate on invariant-theoretic properties of degenerations of isotropy representations involved. To this end, we develop some theory on the corresponding CSS and on the triples of commuting involutions containing conjugate involutions.

A dyad is a pair of different commuting conjugate involutions. Any dyad gives rise to a quaternionic decomposition with additional "symmetry". For a fixed $\sigma_{1}$, we provide a universal construction of dyads $\left\{\sigma_{1}, \sigma_{2}\right\}$, using automorphisms of order 4 (Prop. 2.5). This implies that every involution can be a member of a dyad. We also describe all possible conjugacy classes of involutions $\sigma_{3}=\sigma_{1} \sigma_{2}$ via the restricted root system of $G / G^{\sigma_{1}}$.

or $\sigma_{3}$. Let $\mathfrak{c}_{\alpha}$ be a CSS of $\mathfrak{g}_{\alpha}$ (i.e., $\mathfrak{c}_{\alpha}$ is a little CSS). Then $\mathfrak{c}_{\alpha}$ is contained in a CSS of $\mathfrak{g}_{\alpha} \oplus \mathfrak{g}_{\gamma}$ or $\mathfrak{g}_{\alpha} \oplus \mathfrak{g}_{\beta}$ (i.e., of the (-1)-eigenspaces of two big involutions). If at least one such embedding appears to be an equality, then we call it a coincidence of Cartan subspaces. Clearly, there are totally six possibilities for such coincidences. This can also be expressed as the equality of ranks for certain little and big symmetric spaces. We prove two sufficient conditions for a coincidence of CSS:

- If $\sigma_{1}$ is an involution of maximal rank, then $\mathfrak{c}_{10}$ is also a CSS in $\mathfrak{g}_{01} \oplus \mathfrak{g}_{10}$ and $\mathfrak{c}_{11}$ is also a CSS in $\mathfrak{g}_{01} \oplus \mathfrak{g}_{11}$ (Theorem 3.3).

- If $\left\{\sigma_{1}, \sigma_{2}\right\}$ is a dyad, then $\mathfrak{c}_{11}$ is a CSS in both $\mathfrak{g}_{01} \oplus \mathfrak{g}_{11}$ and $\mathfrak{g}_{10} \oplus \mathfrak{g}_{11}$ (Theorem 3.7).

Let $G_{0 \star}$ denote the connected subgroup of $G$ with Lie algebra $\mathfrak{g}_{0 \star}:=\mathfrak{g}_{00} \oplus \mathfrak{g}_{01}$, and $\mathfrak{g}_{1 \star}:=\mathfrak{g}_{10} \oplus \mathfrak{g}_{11}$. Then $\left(G_{0 \star} \mathfrak{g}_{1 \star}\right)$ is the isotropy representation associated with the (big) symmetric space $G / G_{0 \star}$. We observe that each big symmetric space of $G$ admits two degenerations to symmetric spaces of non-reductive algebraic groups; accordingly, each big 
isotropy representation also admits two degenerations. The corresponding non-reductive Lie algebras are $\mathbb{Z}_{2}$-contractions of $\mathfrak{g}$ in the sense of $[16,17]$. Let $\mathfrak{g}\left\langle\sigma_{i}\right\rangle$ be the $\mathbb{Z}_{2}$-contraction of $\mathfrak{g}$ determined by $\sigma_{i}$. For instance, $\mathfrak{g}\left\langle\sigma_{2}\right\rangle=\mathfrak{g}_{\star 0} \ltimes \mathfrak{g}_{\star 1}^{a}$, where $\mathfrak{g}_{\star 0}=\mathfrak{g}_{00} \oplus \mathfrak{g}_{10}$ and $\mathfrak{g}_{\star 1}=\mathfrak{g}_{01} \oplus \mathfrak{g}_{11}$ are the eigenspaces of $\sigma_{2}$, and the superscript ' $a$ ' means that $\mathfrak{g}_{\star 1}$ is regarded an abelian ideal. The corresponding connected group is $G\left\langle\sigma_{2}\right\rangle=G_{\star 0} \ltimes \exp \left(\mathfrak{g}_{\star 1}^{a}\right)$. Remarkably, both $\sigma_{1}$ and $\sigma_{3}$ remain involutions of the group $G\left\langle\sigma_{2}\right\rangle$ and algebra $\mathfrak{g}\left\langle\sigma_{2}\right\rangle$. Then $\mathfrak{g}\left\langle\sigma_{2}\right\rangle^{\sigma_{1}}=\mathfrak{g}_{00} \ltimes \mathfrak{g}_{01}^{a}=: \mathfrak{k}$, which is the $\mathbb{Z}_{2}$-contraction of $\mathfrak{g}^{\sigma_{1}}=\mathfrak{g}_{0 \star}$ determined by $\sigma_{2}$, i.e., $\mathfrak{g}\left\langle\sigma_{2}\right\rangle^{\sigma_{1}}=\mathfrak{g}^{\sigma_{1}}\left\langle\sigma_{2}\right\rangle$. The (-1)-eigenspace of $\sigma_{1}$ in $\mathfrak{g}\left\langle\sigma_{2}\right\rangle$ is the k-module denoted by $\mathfrak{g}_{10} \propto \mathfrak{g}_{11}$. Likewise, starting with the $\mathbb{Z}_{2}$-contraction $\mathfrak{g}\left\langle\sigma_{3}\right\rangle$, we still obtain $\mathfrak{g}\left\langle\sigma_{3}\right\rangle^{\sigma_{1}}=\mathfrak{k}$, whereas the $(-1)$-eigenspace of $\sigma_{1}$ in $\mathfrak{g}\left\langle\sigma_{3}\right\rangle$ is the $\mathfrak{k}$-module denoted by $\mathfrak{g}_{11} \propto \mathfrak{g}_{10}$.

Let $G_{00}$ be the connected subgroup of $G$ with Lie algebra $\mathfrak{g}_{00}$ and $N_{01}:=\exp \left(\mathfrak{g}_{01}^{a}\right)$. Then $K=G_{00} \ltimes N_{01}$ is the identity component of both $G\left\langle\sigma_{2}\right\rangle^{\sigma_{1}}$ and $G\left\langle\sigma_{3}\right\rangle^{\sigma_{1}}$, and $\operatorname{Lie}(K)=\mathfrak{k}$. The symmetric spaces $G\left\langle\sigma_{2}\right\rangle / K$ and $G\left\langle\sigma_{3}\right\rangle / K$ can be regarded as degenerations of $G / G_{0 \star}$, and we prove that $G\left\langle\sigma_{2}\right\rangle / K$ and $G\left\langle\sigma_{3}\right\rangle / K$ are affine varieties. The $K$-modules $\mathfrak{g}_{10} \propto \mathfrak{g}_{11}$ and $\mathfrak{g}_{11} \propto \mathfrak{g}_{10}$ have the same underlying vector space $\mathfrak{g}_{1 \star}$ and afford the same action of the reductive subgroup $G_{00} \subset K$. But the actions of the unipotent radical $N_{01} \subset K$ are different (see Section 4 for precise formulae). We show that $V:=\mathfrak{g}_{10} \propto \mathfrak{g}_{11}$ and $V^{*}:=\mathfrak{g}_{11} \propto \mathfrak{g}_{10}$ are dual $K$-modules. Thus, the isotropy representations $(K: V)$ and $\left(K: V^{*}\right)$ are different degenerations of $\left(G_{0 \star}: \mathfrak{g}_{1 \star}\right)$.

Altogether, there are six degenerations of three big isotropy representations, and we study invariant-theoretic properties of these degenerated isotropy representations. This generalises the setting of [17], where the coadjoint representation of $\mathbb{Z}_{2}$-contractions is studied. The main idea behind our considerations is that, although $K$ is non-reductive, $(K: V)$ retains many good invariant-theoretic properties of $\left(G_{0 \star}: \mathfrak{g}_{1 \star}\right)$. Coincidences of CSS fit in this setting as follows. Suppose that $\mathfrak{c}_{10}$ is also a CSS in $\mathfrak{g}_{10} \oplus \mathfrak{g}_{11}$. Then $\mathbb{F}[V]^{N_{01}} \simeq \mathbb{F}\left[\mathfrak{g}_{10}\right]$, $\mathbb{F}[V]^{K} \simeq \mathbb{F}\left[\mathfrak{g}_{10}\right]^{G_{00}}$, and $\mathbb{F}[V]$ is a free $\mathbb{F}[V]^{K}$-module (Theorem 4.5). Moreover, if $\sigma_{1}, \sigma_{2}$, and $\sigma_{3}$ are conjugate, then all conceivable coincidences of CSS do occur, and such a simple description of invariants applies to all six degenerated isotropy representations.

Most of the quaternionic decompositions have at least one coincidence of CSS. But this is not always the case, and examples are given in Section 3. In general, we prove that (i) the $K$-module $V$ always has a generic stabiliser, (ii) $\operatorname{trdeg} \mathbb{F}(V)^{K}=\operatorname{trdeg} \mathbb{F}\left(\mathfrak{g}_{1 \star}\right)^{G_{0 \star}}$, and (iii) $\mathbb{F}(V)^{K}$ is the fraction field of $\mathbb{F}[V]^{K}$ (Theorem 4.10). Hence this degeneration does not affect the transcendence degree of fields and algebras of invariants. Furthermore, the algebra $\mathbb{F}[V]^{K}$ is bi-graded and there is a 'contraction method' for obtaining $K$-invariants on $V$ and $V^{*}$ from $\mathbb{F}\left[\mathfrak{g}_{1 \star}\right]^{G_{0 \star}}$. Using this method, we describe $\mathbb{F}[V]^{K}$ under less restrictive assumptions than a coincidence of CSS (Theorem 5.4).

As a by-product of our methods, we prove polynomiality of the algebras of invariant differential operators for many degenerations of big symmetric spaces. We also show that 
results of [5] on 'Invariant differential operators on Grassmann manifolds' can be better understood in the framework of quaternionic decomposition, see Section 6.

Throughout, $G$ is a connected semisimple algebraic group and $\mathfrak{g}=\operatorname{Lie}(G)$.

- $\mathfrak{n}_{\mathfrak{g}}(\mathfrak{a})$ (resp. $\mathfrak{z}_{\mathfrak{g}}(\mathfrak{a})$ ) is the normaliser (resp. centraliser) of a subspace $\mathfrak{a} \subset \mathfrak{g}$.

- the centraliser in $\mathfrak{g}$ of $x \in \mathfrak{g}$ is denoted by $\mathfrak{z}_{\mathfrak{g}}(x)$ or $\mathfrak{g}^{x}$.

- If $X$ is an irreducible variety, then $\mathbb{F}[X]$ is the algebra of regular functions and $\mathbb{F}(X)$ is the field of rational functions on $X$. If $X$ is acted upon by an algebraic group $\mathcal{A}$, then $\mathbb{F}[X]^{\mathcal{A}}$ and $\mathbb{F}(X)^{\mathcal{A}}$ denote the respective $\mathcal{A}$-invariant functions.

- If $\mathbb{F}[X]^{\mathcal{A}}$ is finitely generated, then $X / / \mathcal{A}:=\operatorname{Spec}\left(\mathbb{F}[X]^{\mathcal{A}}\right)$.

\section{Generalities ON INVOLUTIONS AND ISOTROPY REPRESENTATIONS}

Our main object is a connected semisimple algebraic group $G$ with Lie algebra $\mathfrak{g}$. The set of all involutions of $\mathfrak{g}$ is denoted by $\operatorname{lnv}(\mathfrak{g})$. The group of inner automorphisms $\operatorname{lnt}(G) \simeq$ $G / Z(G)$ acts on $\operatorname{lnv}(\mathfrak{g})$ by conjugation. Two involutions are said to be conjugate, if they lie in the same $\operatorname{lnt}(G)$-orbit. If $\sigma \in \operatorname{lnv}(\mathfrak{g})$, then $\mathfrak{g}=\mathfrak{g}_{0} \oplus \mathfrak{g}_{1}$ is the corresponding $\mathbb{Z}_{2}$-grading of $\mathfrak{g}$. That is, $\mathfrak{g}_{i}=\left\{x \in \mathfrak{g} \mid \sigma(x)=(-1)^{i} x\right\}$. We also say that $\left(\mathfrak{g}, \mathfrak{g}_{0}\right)$ is a symmetric pair. Whenever we wish to stress that $\mathfrak{g}_{0}$ and $\mathfrak{g}_{1}$ are determined by $\sigma$, we write $\mathfrak{g}^{\sigma}$ and $\mathfrak{g}_{1}^{(\sigma)}$ for them. We assume that $\sigma$ is induced by an involution of $G$, which is denoted by the same letter. The connected subgroup of $G$ with Lie algebra $\mathfrak{g}_{0}$ is denoted by $G_{0}$, while the fixedpoint subgroup of $\sigma$ is denoted by $G^{\sigma}$. Hence $G_{0}$ is the identity component of $G^{\sigma}$, and $G_{0}=G^{\sigma}$ if $G$ is simply-connected. The representation of $G_{0}$ in $\mathfrak{g}_{1}$, denoted $\left(G_{0}: \mathfrak{g}_{1}\right)$, is the isotropy representation of the symmetric space $G / G_{0}$.

We freely use invariant-theoretic results on the $G_{0}$-action on $\mathfrak{g}_{1}$ or $G / G_{0}$, as exposed in [11],[18]. A Cartan subspace (=CSS) is a maximal subspace of $\mathfrak{g}_{1}$ consisting of pairwise commuting semisimple elements. All CSS of $\mathfrak{g}_{1}$ are $G_{0}$-conjugate and their common dimension is called the rank of the symmetric space $G / G_{0}$, denoted $\operatorname{rk}\left(G / G_{0}\right)$.

The Cartan subspaces are characterised by the following property:

(1.1) Suppose that a subspace $\mathfrak{a} \subset \mathfrak{g}_{1}$ consists of pairwise commuting semisimple elements. Then $\mathfrak{a}$ is a CSS if and only if $\mathfrak{z}_{\mathfrak{g}}(\mathfrak{a}) \cap \mathfrak{g}_{1}=\mathfrak{a}$ [11, Ch. I].

Let $\mathfrak{c}$ be a CSS of $\mathfrak{g}_{1}$. Then every semisimple element of $\mathfrak{g}_{1}$ is $G_{0}$-conjugate to an element of $\mathfrak{c}$ and $G_{0} \cdot \mathfrak{c}$ is dense in $\mathfrak{g}_{1}$. The generalised Weyl group for $\mathfrak{c}, W_{0}$, is defined to be $N_{0}(\mathfrak{c}) / Z_{0}(\mathfrak{c})$, where $N_{0}(\mathfrak{c})=\left\{s \in G_{0} \mid s \cdot \mathfrak{c} \subset \mathfrak{c}\right\}$ and $Z_{0}(\mathfrak{c})=\left\{s \in G_{0} \mid s \cdot x=x \forall x \in \mathfrak{c}\right\}$. An element $x \in \mathfrak{g}_{1}$ is called $G_{0}$-regular if the orbit $G_{0} \cdot x$ is of maximal dimension. Below, we summarise basic invariant-theoretic properties of the isotropy representation:

- Let $x \in \mathfrak{g}_{1}$. The orbit $G_{0} \cdot x$ is closed if and only if $G_{0} \cdot x \cap \mathfrak{c} \neq \varnothing$;

- $x \in \mathfrak{g}_{1}$ is $G_{0}$-regular and semisimple $\Leftrightarrow \mathfrak{z}_{\mathfrak{g}}(x) \cap \mathfrak{g}_{1}$ is a CSS; 
- Each fibre of the quotient morphism $\pi: \mathfrak{g}_{1} \rightarrow \mathfrak{g}_{1} / / G_{0}=\operatorname{Spec}\left(\mathbb{F}\left[\mathfrak{g}_{1}\right]^{G_{0}}\right)$ consists of finitely many $G_{0}$-orbits. The dimension of each fibre equals $\operatorname{dim} \mathfrak{g}_{1}-\operatorname{dim} \mathfrak{c}$.

- The restriction of polynomial functions $\mathbb{F}\left[\mathfrak{g}_{1}\right] \rightarrow \mathbb{F}[\mathfrak{c}]$ induces an isomorphism $\mathbb{F}\left[\mathfrak{g}_{1}\right]^{G_{0}} \stackrel{\sim}{\longrightarrow} \mathbb{F}[\mathfrak{c}]^{W_{0}}$ (Chevalley's restriction theorem);

- $W_{0}$ is a finite reflection group in $G L(\mathfrak{c})$. Hence $\mathbb{F}\left[\mathfrak{g}_{1}\right]^{G_{0}}$ is a polynomial algebra and $\mathfrak{g}_{1} / / G_{0}$ is an affine space of dimension $\operatorname{dim} \mathfrak{c}$;

- $\mathbb{F}\left[\mathfrak{g}_{1}\right]$ is a free $\mathbb{F}\left[\mathfrak{g}_{1}\right]^{G_{0}}$-module.

A torus $S$ of $G$ is $\sigma$-anisotropic, if $\sigma(s)=s^{-1}$ for all $s \in S$. A CSS is the Lie algebra of a maximal $\sigma$-anisotropic torus.

- We say that $\sigma \in \operatorname{lnv}(\mathfrak{g})$ is of maximal rank if $\mathfrak{g}_{1}$ contains a Cartan subalgebra of $\mathfrak{g}$. As is well known, $\operatorname{dim} \mathfrak{g}_{1}-\operatorname{dim} \mathfrak{g}_{0} \leqslant \operatorname{rk} \mathfrak{g}$ for any $\sigma$, and the equality holds if and only if $\sigma$ is of maximal rank.

- Let $\sigma$ be an inner involution of $\mathfrak{g}$. We say that $\sigma$ is quasi-maximal if $\mathfrak{g}_{1}$ contains a regular semisimple element of $\mathfrak{g}$.

Lemma 1.1. Given $\sigma \in \operatorname{Inv}(\mathfrak{g})$, the subspace $\mathfrak{g}_{1}$ contains a regular semisimple element of $\mathfrak{g}$ if and only if it contains a regular nilpotent element of $\mathfrak{g}$.

Proof. For $e \in \mathfrak{g}_{1}$ nilpotent, there is a normal $\mathfrak{s l}_{2}$-triple $\{e, h, f\}$, i.e., such that $e, f \in \mathfrak{g}_{1}$ and $h \in \mathfrak{g}_{0}$ [11, Prop. 4]. If $e$ is regular, then $e+f$ is regular semisimple (conjugate to $h$ ).

Conversely, suppose that $x \in \mathfrak{g}_{1}$ is regular semisimple. Then $G_{0} \cdot x$ is a $G_{0}$-orbit of maximal dimension and there is also a nilpotent element $y \in \mathfrak{g}_{1}$ whose $G_{0}$-orbit has the same dimension. Since $\operatorname{dim} G \cdot z=2 \operatorname{dim} G_{0} \cdot z$ for all $z \in \mathfrak{g}_{1}$ [11, Prop.5], we see that $y$ is regular in $\mathfrak{g}$.

Remark 1.2. It follows from Lemma 1.1 and [15, Theorem 2.3] that the quasi-maximal involutions, as well as involutions of maximal rank, form a single $\operatorname{lnt}(G)$-orbit.

Let $k_{0}$ (resp. $k_{1}$ ) denote the number of even (resp. odd) exponents of $\mathfrak{g}$, so that $k_{0}+k_{1}=$ rk $\mathfrak{g}$.

Proposition 1.3. (i) If $\sigma$ is of maximal rank, then $\mathrm{rk} \mathfrak{g}_{0}=k_{1}$.

(ii) If $\sigma$ is quasi-maximal, then $\operatorname{dim} \mathfrak{g}_{0}-\operatorname{dim} \mathfrak{g}_{1}=k_{0}-k_{1}$ and $\operatorname{dim}\left(\mathfrak{g}_{1} / / G_{0}\right)=k_{1}$.

Proof. Part (i) is proved in [14, Lemma in p. 1473].

In view of Lemma 1.1, part (ii) follows from [15, Theorem 3.3] with $m=2$.

The quasi-maximal involutions and involutions of maximal rank coincide if and only if the Weyl group of $\mathfrak{g}$ contains -1 if and only if all exponents of $\mathfrak{g}$ are odd. The property of having maximal rank is inheritable in the following sense.

Lemma 1.4. Let $x \in \mathfrak{g}_{1}$ be semisimple. If $\sigma$ is of maximal rank, then the restriction of $\sigma$ to $\mathfrak{z}_{\mathfrak{g}}(x)$ and $\left[\mathfrak{z}_{\mathfrak{g}}(x), \mathfrak{z}_{\mathfrak{g}}(x)\right]$ is also of maximal rank. 
Warning. The corresponding assertion for quasi-maximal involutions is not always true.

\section{QUATERNIONIC DECOMPOSITIONS, TRIADS, AND DYADS}

Let $\sigma_{1}$ and $\sigma_{2}$ be different commuting involutions of $\mathfrak{g}$. The corresponding $\mathbb{Z}_{2} \times \mathbb{Z}_{2}$-grading of $\mathfrak{g}$ is:

$$
\mathfrak{g}=\bigoplus_{i, j=0,1} \mathfrak{g}_{i j}, \text { where } \mathfrak{g}_{i j}=\left\{x \in \mathfrak{g} \mid \sigma_{1}(x)=(-1)^{i} x \quad \& \quad \sigma_{2}(x)=(-1)^{j} x\right\}
$$

We also say that it is a quaternionic decomposition of $\mathfrak{g}$ (determined by $\sigma_{1}$ and $\sigma_{2}$ ). Let $\sigma_{3}:=\sigma_{1} \sigma_{2}$. The involutions $\sigma_{1}, \sigma_{2}$, and $\sigma_{3}$ are said to be big. The involutions induced on the fixed-point subalgebras of big involutions are said to be little. The same terminology applies to the corresponding $\mathbb{Z}_{2}$-gradings, CSS, etc. Thus, associated with $(2 \cdot 1)$, one has three big and three little isotropy representations. It is convenient for us to organise the summands of $(2 \cdot 1)$ in a $2 \times 2$ "matrix":

$$
\mathfrak{g}=\begin{array}{c:cc}
\mathfrak{g}_{00} & \mathfrak{g}_{01} \\
\hdashline \mathfrak{g}_{10} & \mathfrak{g}_{01} & \mathfrak{g}_{11}
\end{array} \sigma_{1}
$$

Here the horizontal (resp. vertical) dotted line separates the eigenspaces of $\sigma_{1}$ (resp. $\sigma_{2}$ ), whereas two diagonals of this matrix represent the eigenspaces of $\sigma_{3}$. Hence the first row, first column, and the main diagonal represent the three little $\mathbb{Z}_{2}$-gradings (of $\mathfrak{g}^{\sigma_{1}}, \mathfrak{g}^{\sigma_{2}}$, and $\mathfrak{g}^{\sigma_{3}}$, respectively).

We repeatedly use the following notation for the eigenspaces of $\sigma_{1}$ and $\sigma_{2}$ :

$$
\mathfrak{g}_{0 \star}:=\mathfrak{g}_{00} \oplus \mathfrak{g}_{01}, \mathfrak{g}_{1 \star}:=\mathfrak{g}_{10} \oplus \mathfrak{g}_{11}, \quad \mathfrak{g}_{\star 0}:=\mathfrak{g}_{00} \oplus \mathfrak{g}_{10}, \mathfrak{g}_{\star 1}:=\mathfrak{g}_{01} \oplus \mathfrak{g}_{11} .
$$

Likewise, the connected subgroup of $G$ corresponding to $\mathfrak{g}_{0 \star}$ is denoted by $G_{0 \star}$, etc.

Following Vinberg $[25,0.3]$, we say that a triple $\left\{\sigma_{1}, \sigma_{2}, \sigma_{3}\right\} \subset \operatorname{lnv}(\mathfrak{g})$ is a triad if $\sigma_{1} \sigma_{2}=\sigma_{3}$ and all these involutions are conjugate. A complete classification of triads is obtained in [25, Sect. 3]. Obviously, triads lead to the most "symmetric" quaternionic decompositions. Below, we are interested in more general (hence, less symmetric) decompositions.

Definition 1. We say that $\left\{\sigma_{1}, \sigma_{2}\right\} \subset \operatorname{lnv}(\mathfrak{g})$ is a dyad if $\sigma_{1}, \sigma_{2}$ are conjugate and $\sigma_{1} \sigma_{2}=\sigma_{2} \sigma_{1}$. That is, we do not require that the third involution $\sigma_{3}=\sigma_{1} \sigma_{2}$ is necessarily conjugate to $\sigma_{1}$. Note that the product of two conjugate involutions (not necessarily commuting) is always an inner automorphism of $\mathfrak{g}$. For, if $\sigma_{2}=\operatorname{Int}(g) \cdot \sigma_{1} \cdot \operatorname{Int}\left(g^{-1}\right)$, then $\sigma_{1} \sigma_{2}=\operatorname{Int}\left(\sigma_{1}(g) g^{-1}\right)$. Therefore, any triad consists of inner involutions. A member of a dyad can be an outer involution. But the third involution, $\sigma_{3}$, is necessarily inner.

We begin with describing the dyads containing involutions of maximal rank.

Proposition 2.1. Let $\mu$ be a semisimple inner automorphism of $\mathfrak{g}$. Then there exist involutions of maximal rank $\vartheta$ and $\vartheta^{\prime}$ such that $\mu=\vartheta \vartheta^{\prime}$. 
Proof. By assumption, $\mu=\operatorname{Int}(s)$ for a semisimple element $s \in G$. Choose an involution of maximal rank $\vartheta$ such that $s$ belongs to a $\vartheta$-anisotropic maximal torus $T$. Clearly, the mapping $T \rightarrow T, t \mapsto \vartheta(t) t^{-1}=t^{-2}$ is onto. Therefore, $s=\vartheta(g) g^{-1}$ for some $g \in T$. Set $\vartheta^{\prime}=\operatorname{Int}(g) \cdot \vartheta \cdot \operatorname{Int}\left(g^{-1}\right)$. It is another involution of maximal rank, and a direct computation shows that $\vartheta \vartheta^{\prime}=\operatorname{Int}\left(\vartheta(g) g^{-1}\right)=\mu$.

\section{Proposition 2.2.}

1) Suppose that $\mu \in \operatorname{lnv}(\mathfrak{g})$ is inner. Then there are commuting involutions of maximal rank, $\vartheta$ and $\vartheta^{\prime}$, such that $\mu=\vartheta \vartheta^{\prime}$. Moreover, $\vartheta$ and $\vartheta^{\prime}$ induce an involution of maximal rank of $\mathfrak{g}^{\mu}$.

2) For commuting involutions of maximal rank, $\vartheta$ and $\vartheta^{\prime}$, the following conditions are equivalent:

(i) the inner involution $\mu=\vartheta \vartheta^{\prime}$ is quasi-maximal;

(ii) $\left.\vartheta^{\prime}\right|_{\mathfrak{g}^{\vartheta}}$ is an involution of maximal rank of $\mathfrak{g}^{\vartheta}$;

(iii) $\left.\vartheta\right|_{\mathfrak{g}^{\vartheta^{\prime}}}$ is an involution of maximal rank of $\mathfrak{g}^{\vartheta^{\prime}}$.

Proof. 1) Suppose that $\mu=\operatorname{Int}(s)$. Then $s^{2} \in Z(G)$ and $\operatorname{Int}(s)=\operatorname{Int}\left(s^{-1}\right)$. Choose $\vartheta$ and $\vartheta^{\prime}$ as in the proof of Proposition 2.1. Then $\vartheta \vartheta^{\prime}=\operatorname{Int}(s)$ and $\vartheta^{\prime} \vartheta=\operatorname{Int}\left(s^{-1}\right)$. Thus, $\vartheta$ and $\vartheta^{\prime}$ commute. Consider the quaternionic decomposition of $\mathfrak{g}$ determined by $\left(\vartheta, \vartheta^{\prime}\right)$ :

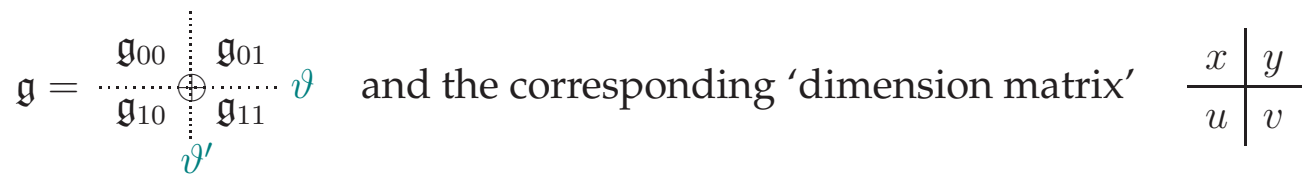

(i.e., $x=\operatorname{dim} \mathfrak{g}_{00}$, etc.). Let $U$ be a maximal unipotent subgroup of $G$. Since $\vartheta$ and $\vartheta^{\prime}$ are of maximal rank,

$$
x+y=x+u=\operatorname{dim} U, \quad y+v=u+v=\operatorname{dim} U+\operatorname{rkg} .
$$

Therefore, $u=y$ and $v-x=\operatorname{rkg}$. Consequently, $\operatorname{dim} \mathfrak{g}_{11}-\operatorname{dim} \mathfrak{g}_{00}=\operatorname{rk} \mathfrak{g}=\operatorname{rk} \mathfrak{g}^{\mu}$. Hence $\mathfrak{g}^{\mu}=\mathfrak{g}_{00} \oplus \mathfrak{g}_{11}$ is a $\mathbb{Z}_{2}$-grading of maximal rank.

2) (i) $\Rightarrow$ (ii),(iii). By the assumption and Proposition 1.3(ii), we have $x+v-2 y=k_{0}-k_{1}$. Consequently, $x=\left(\operatorname{dim} U-k_{1}\right) / 2$ and $y=\left(\operatorname{dim} U+k_{1}\right) / 2$. Hence, $y-x=k_{1}=\operatorname{rk} \mathfrak{g}^{\vartheta}=$ $\mathrm{rk} \mathfrak{g}^{\vartheta^{\prime}}$. Therefore, the induced $\mathbb{Z}_{2}$-grading of $\mathfrak{g}^{\vartheta}$ (or $\left.\mathfrak{g}^{\vartheta^{\prime}}\right)$ is of maximal rank.

Conditions (ii) and (iii) are equivalent, since the dimension matrix is symmetric.

(ii) $\Rightarrow$ (i). By assumption, $\mathfrak{g}_{01}$ contains a Cartan subalgebra of $\mathfrak{g}^{\vartheta}$. Furthermore, the fixedpoint subalgebra of any involution (e.g. $\left.\mathfrak{g}^{\vartheta}\right)$ contains a regular semisimple element $[18$, Lemma 5.3]. Hence $\mathfrak{g}_{01}$ contains a regular semisimple element of $\mathfrak{g}$. Thus, $\mathfrak{g}_{01} \oplus \mathfrak{g}_{10}=\mathfrak{g}_{1}^{(\mu)}$ contains a regular semisimple element of $\mathfrak{g}$, i.e., $\mu$ is quasi-maximal.

Definition 2. A triple $\left\{\vartheta, \vartheta^{\prime}, \mu\right\} \subset \operatorname{lnv}(\mathfrak{g})$ is said to be canonical, if $\vartheta$ and $\vartheta^{\prime}$ are involutions of maximal rank and $\vartheta \vartheta^{\prime}=\mu$ is quasi-maximal. The corresponding quaternionic decomposition is also called canonical. 
Remark 2.3. By Proposition 2.2, all little involutions involved in the canonical decomposition are of maximal rank. Furthermore, $\mathfrak{g}_{11}$ contains a Cartan subalgebra of $\mathfrak{g}$. Another interesting feature is that each of three subspaces $\mathfrak{g}_{1 \star}, \mathfrak{g}_{\star 1}$, and $\mathfrak{g}_{10} \oplus \mathfrak{g}_{01}$ meets all nilpotent $G$-orbits in $\mathfrak{g}$. This follows from results of Antonyan [1]. In Section 5, we obtain further results on canonical decompositions.

As we shall shortly see, every involution is a member of a dyad. This raises the natural question:

What (conjugacy classes of) involutions can occur as products of two members of dyads containing given $\sigma \in \operatorname{lnv}(\mathfrak{g})$ ?

For instance, Proposition 2.2(1) asserts that every inner involution as product from a dyad of involutions of maximal rank. We give below a general answer in terms of the reduced root system for $\sigma$. The following is a recipe for constructing dyads.

Proposition 2.4. For any $\sigma_{1} \in \operatorname{Inv}(\mathfrak{g})$, there is $\phi \in \operatorname{Int}(G)$ such that $\phi^{4}=\mathrm{id}, \sigma_{2}:=\phi \sigma_{1} \phi^{-1}$ commutes with $\sigma_{1}$, and $\sigma_{1} \sigma_{2}=\phi^{2}$. In particular, $\left\{\sigma_{1}, \sigma_{2}\right\}$ is a dyad.

Proof. Let $S$ be a one-dimensional $\sigma_{1}$-anisotropic torus in $G$ and $s \in S$ be an element such that $s^{4} \in Z(G)$. Letting $\phi=\operatorname{Int}(s)$, one has $\phi^{4}=\mathrm{id}, \sigma_{1} \phi \sigma_{1}=\phi^{-1}$, and the remaining relations are easily verified.

Remarkably, all the dyads are obtained in this way!

Proposition 2.5. Suppose that $\left\{\sigma_{1}, \sigma_{2}\right\}$ is a dyad. Then there exists $\phi \in \operatorname{Int}(G)$ such that $\phi^{4}=\mathrm{id}$, $\sigma_{2}:=\phi \sigma_{1} \phi^{-1}$, and $\sigma_{1} \sigma_{2}=\phi^{2}$.

Proof. Suppose that $\sigma_{2}=\operatorname{Int}(g) \sigma_{1} \operatorname{Int}\left(g^{-1}\right)$ for some $g \in G$ and hence $\sigma_{1} \sigma_{2}=\operatorname{Int}\left(\sigma_{1}(g) g^{-1}\right)$. Set $\tilde{g}=\sigma_{1}(g) g^{-1}$. Since $\sigma_{1}$ and $\sigma_{2}$ commute, we have $\tilde{g}^{2} \in Z(G), \sigma_{1}(\tilde{g})=\tilde{g}^{-1}$, and $\sigma_{2}(\tilde{g})=$ $\tilde{g}^{-1}$. By [18, Prop. 6.3], the property that $\tilde{g}$ is semisimple and $\tilde{g}=\sigma_{1}(g) g^{-1}$ guarantees us that $\tilde{g}$ is contained in a $\sigma_{1}$-anisotropic one-dimensional torus. It follows that there exists $s \in G$ such that $s^{2}=\tilde{g}$ and still $\sigma_{1}(s)=s^{-1}$. Then $\phi=\operatorname{Int}(s)$ will do. An easy verification is left to the reader.

Let $C$ be a maximal $\sigma_{1}$-anisotropic torus (hence Lie $(C)$ is a CSS in $\mathfrak{g}_{1 \star}$ ). Recall that a restricted root of $C$ is any non-trivial weight in the decomposition of $\mathfrak{g}$ into the sum of weight spaces of $C$. Write $\Phi^{C}$ for the set of all restricted roots. That is,

$$
\mathfrak{g}=\mathfrak{g}^{C} \oplus\left(\bigoplus_{\gamma \in \Phi^{C}} \mathfrak{g}_{\gamma}\right)
$$

We use the additive notation for the operation in $\mathfrak{X}(C)$, the character group of $C$, and regard $\Phi^{C}$ as a subset of the vector space $\mathfrak{X}(C) \otimes_{\mathbb{Z}} \mathbb{R}$. The set $\Phi^{C}$ satisfies the usual axioms of 
finite root systems [6]. The notable difference from the structure theory of split semisimple Lie algebras is that the root system $\Phi^{C}$ can be non-reduced and that $m_{\gamma}=\operatorname{dim} \mathfrak{g}_{\gamma}$ $\left(\gamma \in \Phi^{C}\right)$ can be greater than 1.

The (universal) construction of dyads described in the proof of Prop. 2.4 means that, for given $\sigma_{1}$, all possible involutions $\sigma_{3}$ are obtained in the following way. Take a onedimensional torus $S \subset C$, consider the corresponding $\mathbb{Z}$-grading of $\mathfrak{g}$ :

$$
\mathfrak{g}=\bigoplus_{i \in \mathbb{Z}} \mathfrak{g}^{S}(i)
$$

and then set $\mathfrak{g}_{0}=\oplus_{i \in \mathbb{Z}} \mathfrak{g}^{S}(2 i)$ and $\mathfrak{g}_{1}=\oplus_{i \in \mathbb{Z}} \mathfrak{g}^{S}(2 i+1)$. Alternatively, this can be expressed as follows. Choose a linear form $\ell$ on $\mathfrak{X}(C) \otimes_{\mathbb{Z}} \mathbb{R}$ that takes integral values on $\Phi^{C}$. (One should assume that $\ell\left(\Phi^{C}\right) \not \subset 2 \mathbb{Z}$.) Define $\Phi_{1}^{C}$ to be the set of all restricted roots $\gamma$ such that $\ell(\gamma)$ is odd. Then $\bigoplus_{\gamma \in \Phi_{1}^{C}} \mathfrak{g}_{\gamma}$ is the $(-1)$-eigenspace of $\sigma_{3}$.

Remark 2.6. If $\Phi^{C}$ is reduced, then all possible involution $\sigma_{3}$ are associated with the inner involutions of the semisimple Lie algebra with root system $\Phi^{C}$.

Example 2.7. If $\sigma_{1}$ is of maximal rank, then $C$ is a maximal torus of $G$ and $\Phi^{C}$ is the usual root system of $\mathfrak{g}$. Here all one-dimensional tori are at our disposal, hence $\sigma_{3}$ can be any inner involution (which is already known from Prop. 2.2).

Example 2.8. Let $\sigma_{1}$ be an involution EIX, i.e., $\mathfrak{g}$ is $\mathbf{E}_{8}$ and $\mathfrak{g}^{\sigma_{1}}$ is $\mathbf{E}_{7} \times \mathbf{A}_{1}$. Here $\operatorname{dim} C=4$ and $\Phi^{C}$ is of type $\mathbf{F}_{4}$; for $\gamma \in \Phi^{C}$, one has $m_{\gamma}=\left\{\begin{array}{ll}8, & \text { if } \gamma \text { short } \\ 1, & \text { if } \gamma \text { long }\end{array}\right.$, see [6, Ch. X, Table 6] or [26, Table 9]. The Lie algebra $\mathbf{F}_{4}$ has two (conjugacy classes of) inner involutions, and this leads to two possibilities for $\sigma_{3}$. Using information on the multiplicities of restricted roots, one easily computes $\operatorname{dim} \mathfrak{g}^{\sigma_{3}}$, which allows us to identify $\sigma_{3}$. The answer is that involution FI (resp. F II) leads to the involution of $\mathfrak{g}$ with $\operatorname{dim} \mathfrak{g}^{\sigma_{3}}=120$ (resp. 136), i.e., $\sigma_{3}$ is either EVIII or EIX.

\section{COMPARING CARTAN SUbSPACES}

As is explained above, the quaternionic decomposition (2.2) embraces six $\mathbb{Z}_{2}$-gradings. In this section, we compare CSS for little and big $\mathbb{Z}_{2}$-gradings.

For $(i j) \neq(00)$, let $\mathfrak{c}_{i j}$ be a CSS of $\mathfrak{g}_{i j}$; that is, a CSS related to the little $\mathbb{Z}_{2}$-grading $\mathfrak{g}_{00} \oplus \mathfrak{g}_{i j}$. There are also CSS for three big involutions:

$$
\mathfrak{c}_{1 \star} \subset \mathfrak{g}_{1 \star}, \mathfrak{c}_{\star 1} \subset \mathfrak{g}_{\star 1}, \mathfrak{c}_{\star, 1-\star} \subset \mathfrak{g}_{\star, 1-\star}:=\mathfrak{g}_{01} \oplus \mathfrak{g}_{10} .
$$

Each little CSS can be included in two big CSS. E.g., because $\mathfrak{g}_{10} \subset \mathfrak{g}_{1 \star}$ and $\mathfrak{g}_{10} \subset \mathfrak{g}_{\star, 1-\star}$, one can choose Cartan subspaces $\mathfrak{c}_{1 \star}$ and $\mathfrak{c}_{\star, 1-\star}$ such that $\mathfrak{c}_{10} \subset \mathfrak{c}_{1 \star}$ and $\mathfrak{c}_{10} \subset \mathfrak{c}_{\star, 1-\star}$. If at least one equality occurs among all such inclusions, then this will be referred to as a coincidence of CSS (for a given quaternionic decomposition). We obtain two sufficient conditions 
for such a coincidence to happen and provide examples of quaternionic decompositions without coincidences of CSS.

We begin with a preparatory result. Let $\kappa$ denote the Killing form on $\mathfrak{g}$. Set $\mathfrak{m}_{i j}=$ $\left[\mathfrak{g}_{i j}, \mathfrak{g}_{i j}\right] \subset \mathfrak{g}_{00}$ for $(i j) \neq(00)$. Clearly, $\mathfrak{m}_{i j}$ is an (algebraic) ideal of the reductive algebraic Lie algebra $\mathfrak{g}_{00}$. Therefore $\mathfrak{m}_{i j}$ is also reductive and $\left.\kappa\right|_{\mathfrak{m}_{i j}}$ is non-degenerate.

Lemma 3.1. Suppose that $\mathfrak{g}_{11}=0$. Then

(i) $\left[\mathfrak{m}_{01}, \mathfrak{g}_{10}\right]=\left[\mathfrak{m}_{10}, \mathfrak{g}_{01}\right]=0$;

(ii) $\kappa\left(\mathfrak{m}_{01}, \mathfrak{m}_{10}\right)=0$ and $\mathfrak{m}_{01} \cap \mathfrak{m}_{10}=\{0\}$;

(iii) $\mathfrak{m}_{10} \oplus \mathfrak{g}_{10}$ and $\mathfrak{m}_{01} \oplus \mathfrak{g}_{01}$ are disjoint ideals of $\mathfrak{g}$.

Proof. Everything follows from the Jacobi identity, the relation $\left[\mathfrak{g}_{10}, \mathfrak{g}_{01}\right]=0$, and the fact that $\left.\kappa\right|_{\mathfrak{m}_{i j}}$ is non-degenerate.

Corollary 3.2. If $\mathfrak{g}_{0 \star}=\mathfrak{g}^{\sigma_{1}}$ does not contain proper ideals of $\mathfrak{g}$ and $\mathfrak{g}_{11}=0$, then $\mathfrak{g}_{01}=0$ as well. (In particular, this applies if $\sigma_{1}$ is of maximal rank.)

Proof. Since $\sigma_{1}$ is non-trivial, we have $\mathfrak{g}_{10} \neq 0$. As $\mathfrak{m}_{01} \oplus \mathfrak{g}_{01}$ is a proper ideal of $\mathfrak{g}$ lying in $\mathfrak{g}_{0 *}$, it must be zero, i.e., $\mathfrak{g}_{01}=0$.

Now, we are in a position to prove our first result on the coincidence of CSS.

Theorem 3.3. Let $\mathfrak{g}$ be a semisimple Lie algebra and $\left\{\sigma_{1}, \sigma_{2}, \sigma_{3}\right\}$ a triple of involutions of $\mathfrak{g}$ such that $\sigma_{1} \sigma_{2}=\sigma_{3}$. Suppose that $\sigma_{1}$ is of maximal rank. Then (1) any CSS $\mathfrak{c}_{11} \subset \mathfrak{g}_{11}$ is also a CSS in $\mathfrak{g}_{* 1}$, i.e., for $\sigma_{2}$; (2) any CSS $\mathfrak{c}_{10} \subset \mathfrak{g}_{10}$ is also a CSS in $\mathfrak{g}_{10} \oplus \mathfrak{g}_{01}$, i.e., for $\sigma_{3}$.

Proof. Obviously, (2) is obtained from (1) if we permute $\sigma_{2}$ and $\sigma_{3}$. Therefore, it suffices to prove the first assertion.

Set $\mathfrak{l}=\mathfrak{z}_{\mathfrak{g}}\left(\mathfrak{c}_{11}\right)$ and $\mathfrak{s}:=[\mathfrak{l}, \mathfrak{l}]$. Then $\mathfrak{l}$ is a $\left(\sigma_{1}, \sigma_{2}\right)$-stable Levi subalgebra of $\mathfrak{g}$. Let $\mathfrak{z}$ be the centre of $\mathfrak{l}$, so that $\mathfrak{l}=\mathfrak{s} \oplus \mathfrak{z}$. By construction, $\mathfrak{c}_{11} \subset \mathfrak{z}_{11}$. Furthermore, since $\mathfrak{c}_{11}$ is a CSS of $\mathfrak{g}_{11}$, we have $\mathfrak{z}_{\mathfrak{g}}\left(\mathfrak{c}_{11}\right) \cap \mathfrak{g}_{11}=\mathfrak{c}_{11}$, i.e., $\mathfrak{s}_{11}=0$ and $\mathfrak{c}_{11}=\mathfrak{z}_{11}$.

As $\sigma_{1}$ is of maximal rank, there exists a Cartan subalgebra $\mathfrak{t} \subset \mathfrak{g}$ such that $\mathfrak{c}_{11} \subset \mathfrak{t} \subset \mathfrak{g}_{1 \star}$. Then $[\mathfrak{z}, \mathfrak{t}]=0$ and therefore $\mathfrak{z} \subset \mathfrak{t} \subset \mathfrak{g}_{1 \star}$. By Lemma 1.4, the restriction of $\sigma_{1}$ to the semisimple algebra $\mathfrak{s}$ is still of maximal rank. Applying Corollary 3.2 to $\mathfrak{s}$ in place of $\mathfrak{g}$, we conclude that $\mathfrak{s}_{01}=0$. Because $\mathfrak{z} \subset \mathfrak{g}_{1 \star}$, this also means that $\mathfrak{l}_{01}=0$. Thus,

$$
\mathfrak{z}_{\mathfrak{g}}\left(\mathfrak{c}_{11}\right) \cap \mathfrak{g}_{\star 1}=\mathfrak{z}_{\mathfrak{g}}\left(\mathfrak{c}_{11}\right) \cap \mathfrak{g}_{11}=\mathfrak{c}_{11},
$$

which is exactly what we need, in view of $(1 \cdot 1)$.

Alternatively, coincidences of CSS in Theorem 3.3 can be expressed in terms of the rank of symmetric spaces.

Corollary 3.4. If $\vartheta$ and $\sigma$ are commuting involutions and $\vartheta$ is of maximal rank, then 


$$
\operatorname{rk}\left(G / G^{\sigma}\right)=\operatorname{rk}\left(G^{\vartheta \sigma} / G^{\vartheta} \cap G^{\sigma}\right) \text { and } \operatorname{rk}\left(G / G^{\vartheta \sigma}\right)=\operatorname{rk}\left(G^{\sigma} / G^{\vartheta} \cap G^{\vartheta \sigma}\right) \text {. }
$$

The following readily follows from the symmetry between $\sigma_{1}$ and $\sigma_{2}$ :

Corollary 3.5. Suppose that both $\sigma_{1}$ and $\sigma_{2}$ are of maximal rank. Then

(i) any CSS $\mathfrak{c}_{11} \subset \mathfrak{g}_{11}$ is also a CSS in $\mathfrak{g}_{1 \star}$ or in $\mathfrak{g}_{\star 1}$, i.e., for $\sigma_{1}$ or $\sigma_{2}$;

(ii) any CSS $\mathfrak{c}_{01} \subset \mathfrak{g}_{01}$ is also a CSS in $\mathfrak{g}_{10} \oplus \mathfrak{g}_{01}$, i.e., for $\sigma_{3}$;

(iii) any CSS $\mathfrak{c}_{10} \subset \mathfrak{g}_{10}$ is also a CSS in $\mathfrak{g}_{10} \oplus \mathfrak{g}_{01}$, i.e., for $\sigma_{3}$.

Remark 3.6. If both $\sigma_{1}$ and $\sigma_{2}$ are of maximal rank, then $\mathfrak{c}_{11}$ is a Cartan subalgebra of $\mathfrak{g}$ (use Prop. 2.2(1)). This provides another explanation for part (i) in Corollary 3.5.

Our second result on the coincidence of CSS concerns arbitrary dyads.

Theorem 3.7. Let $\mathfrak{g}$ be a semisimple Lie algebra and $\left\{\sigma_{1}, \sigma_{2}, \sigma_{3}\right\}$ a triple of involutions of $\mathfrak{g}$ such that $\sigma_{1} \sigma_{2}=\sigma_{3}$. Suppose that $\left\{\sigma_{1}, \sigma_{2}\right\}$ is a dyad. Then any CSS $\mathfrak{c}_{11} \subset \mathfrak{g}_{11}$ is also a CSS in $\mathfrak{g}_{1 \star}$ or $\mathfrak{g}_{\star 1}$, i.e., for $\sigma_{1}$ or $\sigma_{2}$;

Proof. As in the proof of Theorem 3.3, we consider the $\left(\sigma_{1}, \sigma_{2}\right)$-stable Levi subalgebra

$$
\mathfrak{z}_{\mathfrak{g}}\left(\mathfrak{c}_{11}\right)=: \mathfrak{l}=\mathfrak{s} \oplus \mathfrak{z},
$$

where $\mathfrak{s}$ is semisimple and $\mathfrak{z}$ is the centre of $\mathfrak{l}$. Since $\mathfrak{c}_{11}$ is a CSS in $\mathfrak{g}_{11}$, we have $\mathfrak{l}_{11}=\mathfrak{c}_{11}(=$ $\left.\mathfrak{z}_{11}\right)$, so that $\mathfrak{s}_{11}=\{0\}$, and our task is to prove that $\mathfrak{l}_{01}=\mathfrak{l}_{10}=\{0\}$.

Since $\sigma_{1}$ and $\sigma_{2}$ are conjugate, $\sigma_{2}=\operatorname{Int}(g) \sigma_{1} \operatorname{Int}\left(g^{-1}\right)$ for some $g \in G$. Then $\sigma_{3}=\operatorname{Int}(\tilde{g})$, where $\tilde{g}=\sigma_{1}(g) g^{-1}$. Since $\sigma_{1} \sigma_{2}=\sigma_{2} \sigma_{1}$ and $\operatorname{Int}(\tilde{g})=\operatorname{Int}\left(\tilde{g}^{-1}\right)$, we also have $\tilde{g}=g^{-1} \sigma_{2}(g)$. The choice of $g$ is not unique, and we are going to demonstrate that $g$ can be chosen to have some extra properties.

Let $x \in \mathfrak{c}_{11}$ be generic. In particular, $x \in \mathfrak{g}^{\sigma_{3}}$, i.e., $\operatorname{Int}(\tilde{g}) \cdot x=x$. Making use of two expressions for $\tilde{g}$, one easily computes that

$$
\sigma_{1}\left(\operatorname{Int}\left(g^{-1}\right) \cdot x\right)=-\operatorname{Int}\left(g^{-1}\right) \cdot x \text { and } \quad \sigma_{2}(\operatorname{Int}(g) \cdot x)=-\operatorname{Int}(g) \cdot x .
$$

In particular,

$$
\operatorname{Int}\left(g^{-1}\right) \cdot x \subset \mathfrak{g}_{1 \star}=\mathfrak{g}_{1}^{\left(\sigma_{1}\right)} \text { and } \operatorname{Int}(g) \cdot x \subset \mathfrak{g}_{\star 1}=\mathfrak{g}_{1}^{\left(\sigma_{2}\right)} .
$$

Recently, M. Bulois [3, Prop. 6.6] noticed that any $\mathbb{Z}_{2}$-grading $\mathfrak{g}=\mathfrak{g}_{0} \oplus \mathfrak{g}_{1}$ has the following property:

$$
\text { If } x \in \mathfrak{g}_{1} \text { is semisimple, then } G \cdot x \cap \mathfrak{g}_{1}=G_{0} \cdot x .
$$

Applying this, say, to the first inclusion in (3.1) shows that the identity component of $G^{\sigma_{1}}$ contains $p$ such that $\operatorname{Int}\left(g^{-1}\right) \cdot x=\operatorname{Int}(p) \cdot x$, i.e., $g p \in Z_{G}(x)$, the centraliser of $x$ in $G$.

Replacing $g$ with $g p$ does not affect $\tilde{g}$ and $\sigma_{2}$. Therefore, we may assume that our initial $g$ lies in $Z_{G}(x)$. Note that this group is always connected. So far, we did not use the assumption that $x$ is generic in $\mathfrak{c}_{11}$. For generic $x$, we have $\mathfrak{z}_{\mathfrak{g}}(x)=\mathfrak{z}_{\mathfrak{g}}\left(\mathfrak{c}_{11}\right)$. Hence 
$\operatorname{Lie}\left(Z_{G}(x)\right)=\mathfrak{l}$, and we will write $L$ in place of $Z_{G}(x)$. Since $g \in L$, the involutions $\left.\sigma_{1}\right|_{\mathfrak{s}}$ and $\left.\sigma_{2}\right|_{\mathfrak{s}}$ are conjugate with respect to $\operatorname{Int}(S)$, where $S:=(L, L)$ is connected and semisimple. Note that $\operatorname{Lie}(S)=\mathfrak{s}$.

Let us prove that $\mathfrak{s}_{01}=\mathfrak{s}_{10}=\{0\}$. Since $\mathfrak{s}_{11}=\{0\}$, it follows from Lemma 3.1(iii) that $\mathfrak{q}_{10}=\mathfrak{s}_{10} \oplus\left[\mathfrak{s}_{10}, \mathfrak{s}_{10}\right]$ and $\mathfrak{q}_{01}=\mathfrak{s}_{01} \oplus\left[\mathfrak{s}_{01}, \mathfrak{s}_{01}\right]$ are disjoint ideals of $\mathfrak{s}$. Thus, $\mathfrak{s}^{\sigma_{1}} \supset \mathfrak{q}_{01}, \mathfrak{s}^{\sigma_{2}} \supset \mathfrak{q}_{10}$, and $\mathfrak{s}^{\sigma_{1}}$ and $\mathfrak{s}^{\sigma_{1}}$ are conjugate with respect to $S$. It is only possible if $\mathfrak{q}_{10}=\mathfrak{q}_{01}=\{0\}$, i.e., $\mathfrak{s}_{10}=\mathfrak{s}_{01}=\{0\}$. This completes the first part of our programme.

The second part deals with the centre of $\mathfrak{l}$. Let $\mathfrak{t}$ be a Cartan subalgebra of $\mathfrak{g}^{\sigma_{3}}=\mathfrak{g}_{00} \oplus \mathfrak{g}_{11}$ that contains $\mathfrak{c}_{11}$. Since $\sigma_{3}$ is inner, $\mathfrak{t}$ is actually a Cartan subalgebra of $\mathfrak{g}$. As $\mathfrak{t} \subset \mathfrak{z}_{\mathfrak{g}}(x), \mathfrak{t}$ must contain $\mathfrak{z}$. Therefore, $\mathfrak{z} \subset \mathfrak{g}^{\sigma_{3}}$ and $\mathfrak{z}_{01}=\mathfrak{z}_{10}=\{0\}$.

We have proved that $\mathfrak{l}_{01}=\mathfrak{l}_{10}=\{0\}$. Hence $\mathfrak{z}_{\mathfrak{g}}\left(\mathfrak{c}_{11}\right) \cap \mathfrak{g}_{1 \star}=\mathfrak{z}_{\mathfrak{g}}\left(\mathfrak{c}_{11}\right) \cap \mathfrak{g}_{\star 1}=\mathfrak{c}_{11}$, and we are done.

Corollary 3.8. If $\left\{\sigma_{1}, \sigma_{2}, \sigma_{3}\right\}$ is a triad, then every little CSS is also a big CSS in two possible ways. (That is, six coincidences of CSS occur.)

Again, the coincidence of CSS can be expressed in terms of ranks of symmetric spaces:

Corollary 3.9. If $\left\{\sigma_{1}, \sigma_{2}\right\}$ is a dyad, then $\operatorname{rk}\left(G^{\sigma_{1} \sigma_{2}} / G^{\sigma_{1}} \cap G^{\sigma_{2}}\right)=\operatorname{rk}\left(G / G^{\sigma_{1}}\right)$.

Theorems 3.3 and 3.7 show that, for many triples of commuting involutions, there is a coincidence of CSS. However, this is not always the case, and we provide below two examples of commuting triples without coincidences of CSS.

Example 3.10. Let $\mathfrak{g}$ be a semisimple Lie algebra and $\sigma$ an involution of $\mathfrak{g}$ with the corresponding $\mathbb{Z}_{2}$-grading $\mathfrak{g}=\mathfrak{g}_{0} \oplus \mathfrak{g}_{1}$. We specify the requirements on $\sigma$ below. Set $\tilde{\mathfrak{g}}=\mathfrak{g} \oplus \mathfrak{g}$ and define three involutions of $\tilde{\mathfrak{g}}$ as follows:

$$
\sigma_{1}\left(x_{1}, x_{2}\right)=\left(x_{2}, x_{1}\right), \quad \sigma_{2}\left(x_{1}, x_{2}\right)=\left(\sigma\left(x_{1}\right), \sigma\left(x_{2}\right)\right), \quad \sigma_{3}=\sigma_{1} \sigma_{2} .
$$

Then $\tilde{\mathfrak{g}}^{\sigma_{1}}=\Delta(\mathfrak{g})$, the diagonal; $\tilde{\mathfrak{g}}^{\sigma_{2}}=\mathfrak{g}_{0} \oplus \mathfrak{g}_{0} ; \tilde{\mathfrak{g}}^{\sigma_{3}}=\{(x, \sigma(x)) \mid x \in \mathfrak{g}\}$.

Set $\Delta_{-}(M):=\{(m,-m) \mid m \in M\}$ for any subspace $M \subset \mathfrak{g}$. Then the corresponding quaternionic decomposition is:

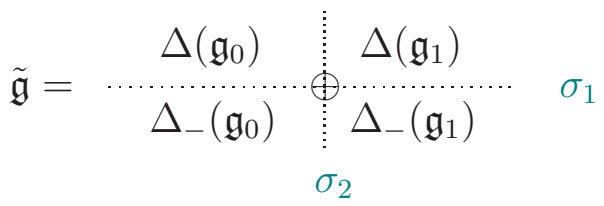

Let $\mathfrak{c}$ be a CSS of $\mathfrak{g}_{1}$ and $\operatorname{dim} \mathfrak{c}=r$. Let $\mathfrak{t}$ be a Cartan subalgebra of $\mathfrak{g}$ and $\mathfrak{t}_{0}$ a Cartan subalgebra of $\mathfrak{g}_{0}$. Both little and big CSS can explicitly be described. We have

$$
\mathfrak{c}_{01}=\Delta(\mathfrak{c}) \subset \Delta\left(\mathfrak{g}_{1}\right), \mathfrak{c}_{10}=\Delta_{-}\left(\mathfrak{t}_{0}\right) \subset \Delta_{-}\left(\mathfrak{g}_{0}\right), \mathfrak{c}_{11}=\Delta_{-}(\mathfrak{c}) \subset \Delta_{-}\left(\mathfrak{g}_{1}\right)
$$

and

$\mathfrak{c}_{1 \star}=\Delta_{-}(\mathfrak{t}) \subset \Delta_{-}(\mathfrak{g}), \mathfrak{c}_{\star 1}=\mathfrak{c} \oplus \mathfrak{c} \subset \mathfrak{g}_{1} \oplus \mathfrak{g}_{1}, \mathfrak{c}_{\star, 1-\star}=\{t,-\sigma(t) \mid t \in \mathfrak{t}\} \subset\{x,-\sigma(x) \mid x \in \mathfrak{g}\}$. 
The absence of coincidences means that each big CSS has strictly bigger dimension than either of two little CSS that can belong to it. This yields the following conditions:

$$
\begin{gathered}
r=\operatorname{dim} \mathfrak{c}_{11}<\operatorname{dim} \mathfrak{c}_{1 \star}=\operatorname{rk} \mathfrak{g}, \quad \operatorname{rk} \mathfrak{g}_{0}=\operatorname{dim} \mathfrak{c}_{10}<\operatorname{dim} \mathfrak{c}_{1 \star}=\operatorname{rk} \mathfrak{g}, \\
r=\operatorname{dim} \mathfrak{c}_{11}<\operatorname{dim} \mathfrak{c}_{\star 1}=2 r, \quad r=\operatorname{dim} \mathfrak{c}_{01}<\operatorname{dim} \mathfrak{c}_{\star 1}=2 r, \\
\operatorname{rk} \mathfrak{g}_{0}=\operatorname{dim} \mathfrak{c}_{10}<\operatorname{dim} \mathfrak{c}_{\star, 1-\star}=\operatorname{rkg}, \quad r=\operatorname{dim} \mathfrak{c}_{01}<\operatorname{dim} \mathfrak{c}_{\star, 1-\star}=\operatorname{rk} \mathfrak{g} .
\end{gathered}
$$

All this amounts to the inequalities $\operatorname{rk} \mathfrak{g}_{0}<\operatorname{rk} \mathfrak{g}$ and $r<\operatorname{rk} \mathfrak{g}$. Both are satisfied if and only if $\sigma$ is outer and not of maximal rank. E.g., such an involution $\sigma$ exists if $\mathfrak{g}$ is a simple Lie algebra of type $\mathbf{A}_{2 n+1}$ or $\mathbf{D}_{2 n+1}$ or $\mathbf{E}_{6}$.

A drawback of Example 3.10 is that $\tilde{\mathfrak{g}}$ is not simple. Actually, it is not easy to discover such an example for simple Lie algebras. A coincidence of some little and big CSS is rather a rule, than exception. For instance, any triple of commuting involutions of exceptional Lie algebras contains either a dyad or an involution of maximal rank, see [9, Table 1]. We can also prove that there is always a coincidence of CSS for the triples of commuting involutions of $\mathfrak{g}=\mathfrak{s l}_{n}$. The following example concerns algebras of type $\mathbf{D}_{N}$.

Example 3.11. $\mathfrak{g}=\mathfrak{s o}_{2 N}$ and $G=S O_{2 N}$. Consider three involutions of $\mathfrak{g}$ with the following fixed-point subalgebras:

$\sigma_{1}, \sigma_{2}: \mathfrak{g l}_{N} ; \quad \sigma_{3}: \mathfrak{s o}_{2 n} \oplus \mathfrak{s o}_{2 m}, m+n=N$.

For $N$ even, $\operatorname{Int}(G)$ contains two conjugacy classes of involutions with the fixed-point subalgebra $\mathfrak{g l}_{N}$, and we will arrange that $\sigma_{1}, \sigma_{2}$ belong to different conjugacy classes. Namely, assume that $\mathfrak{g}$ is represented by the matrices of order $2 N$ that are skew-symmetric w.r.t. the antidiagonal and define the $\sigma_{i}{ }^{\prime}$ s using certain diagonal matrices, as follows:

$$
\begin{aligned}
& \sigma_{1}=\operatorname{Int}(\operatorname{diag}(\underbrace{i, \ldots, i}_{N}, \underbrace{-i, \ldots,-i}_{N})) ; \\
& \sigma_{2}=\operatorname{Int}(\operatorname{diag}(\underbrace{i, \ldots, i}_{m}, \underbrace{-i, \ldots,-i}_{n}, \underbrace{i, \ldots, i}_{n}, \underbrace{-i, \ldots,-i}_{m})) ; \\
& \sigma_{3}=\sigma_{1} \sigma_{2}=\operatorname{Int}(\operatorname{diag}(\underbrace{-1, \ldots,-1}_{m}, \underbrace{1, \ldots, 1}_{n}, \underbrace{1, \ldots, 1}_{n}, \underbrace{-1, \ldots,-1}_{m})) .
\end{aligned}
$$

Here $i=\sqrt{-1}$. One easily presents this quaternionic decomposition in the matrix form. Taking the eigenspaces of $\sigma_{1}$ and $\sigma_{2}$ partition the matrices in $\mathfrak{g}$ in 16 blocks, and we indicate the subspace $\mathfrak{g}_{i j}$ which each block belongs to:

$$
\mathfrak{g}=\left(\begin{array}{llll}
\mathfrak{g}_{00} & \mathfrak{g}_{01} & \mathfrak{g}_{10} & \mathfrak{g}_{11} \\
\mathfrak{g}_{01} & \mathfrak{g}_{00} & \mathfrak{g}_{11} & \mathfrak{g}_{10} \\
\mathfrak{g}_{10} & \mathfrak{g}_{11} & \mathfrak{g}_{00} & \mathfrak{g}_{01} \\
\mathfrak{g}_{11} & \mathfrak{g}_{10} & \mathfrak{g}_{01} & \mathfrak{g}_{00}
\end{array}\right)
$$

The diagonal blocks consists of square matrices of order $m, n, n, m$, respectively. Hence the framed block consists of rectangular matrices of shape $n \times m$. Then one computes that 
$\operatorname{dim} \mathfrak{c}_{01}=\operatorname{dim} \mathfrak{c}_{10}=\min \{n, m\}, \operatorname{dim} \mathfrak{c}_{11}=[n / 2]+[m / 2] ; \quad$ and $\operatorname{dim} \mathfrak{c}_{1 \star}=\operatorname{dim} \mathfrak{c}_{\star 1}=[n+m / 2], \operatorname{dim} \mathfrak{c}_{\star, 1-\star}=2 \min \{n, m\}$.

If $n, m$ are odd, then $\sigma_{1}, \sigma_{2}$ are not conjugate. Furthermore, if $n, m$ are odd and $n \neq m$, then there is no coincidence of CSS, since $[n / 2]+[m / 2]<[n+m / 2]$ and $\min \{n, m\}<$ $[n+m / 2]$.

Remark 3.12. A coincidence of Cartan subspaces has the following invariant-theoretic meaning. Suppose that $\mathfrak{c}_{10} \subset \mathfrak{g}_{10}$ is also a CSS in $\mathfrak{g}_{10} \oplus \mathfrak{g}_{11}=\mathfrak{g}_{1 \star}$. Then, in view of Chevalley's restriction theorem, the natural restriction homomorphism

$$
\varrho: \mathbb{F}\left[\mathfrak{g}_{1 \star}\right]^{G_{0 \star}} \rightarrow \mathbb{F}\left[\mathfrak{g}_{10}\right]^{G_{00}}
$$

is injective, and it makes $\mathbb{F}\left[\mathfrak{g}_{10}\right]^{G_{00}}$ a finite $\mathbb{F}\left[\mathfrak{g}_{1 \star}\right]^{G_{0 \star}}$-module. The degree of the finite ring extension equals the ratio of orders of the corresponding generalised Weyl groups. It should be noted that $\mathbb{F}\left[\mathfrak{g}_{10}\right]^{G_{00}}$ is always a finite $\varrho\left(\mathbb{F}\left[\mathfrak{g}_{1 \star}\right]^{G_{0 \star}}\right)$-module. That is, the point is that such a coincidence of CSS implies the injectivity of $\varrho$.

Putting this in the geometric form, we obtain the commutative diagram

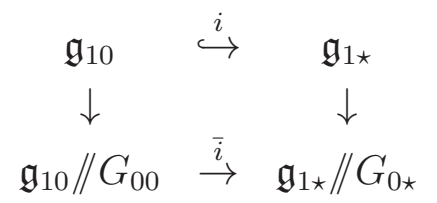

where the vertical arrows are quotient morphisms, $i$ is the embedding, and the morphism $\bar{i}$ is finite and surjective.

\section{DEGENERATIONS OF SYMMETRIC SPACES AND ISOTROPY REPRESENTATIONS}

For any $\sigma \in \operatorname{Inv}(\mathfrak{g})$ with $\mathbb{Z}_{2}$-grading $\mathfrak{g}=\mathfrak{g}_{0} \oplus \mathfrak{g}_{1}$, there is a non-reductive Lie algebra, which is a contraction of $\mathfrak{g}$. Namely, the semi-direct product $\mathfrak{g}\langle\sigma\rangle=\mathfrak{g}_{0} \ltimes \mathfrak{g}_{1}^{a}$ is called a $\mathbb{Z}_{2}$-contraction of $\mathfrak{g}$ (with respect to $\sigma$ ). Here the superscript ' $a$ ' means that the $\mathfrak{g}_{0}$-module $\mathfrak{g}_{1}$ is regarded as an abelian ideal of $\mathfrak{g}\langle\sigma\rangle$. Then $\mathfrak{g}_{1}^{a}$ is also the nilpotent radical of $\mathfrak{g}\langle\sigma\rangle$. The corresponding connected group $G\langle\sigma\rangle$ is a semi-direct product of $G_{0}$ and the abelian unipotent radical $\exp \left(\mathfrak{g}_{1}^{a}\right)$, i.e., $G\langle\sigma\rangle=G_{0} \ltimes \exp \left(\mathfrak{g}_{1}^{a}\right)$.

Invariant-theoretic properties of the adjoint and coadjoint representations of $G\langle\sigma\rangle$ have been studied in $[16,17]$. By [16, Thm. 6.2], the algebra $\mathbb{F}[\mathfrak{g}\langle\sigma\rangle]^{G\langle\sigma\rangle}$ is always polynomial. In [17], it is proved that the algebra $\mathbb{F}\left[\mathfrak{g}\langle\sigma\rangle^{*}\right]^{G\langle\sigma\rangle}$ is polynomial in many cases. There is also a useful method of "contraction" of $G$-invariants. Namely, to any homogeneous $f \in \mathbb{F}[\mathfrak{g}]^{G}$ one can associate an element of either $\mathbb{F}[\mathfrak{g}\langle\sigma\rangle]^{G\langle\sigma\rangle}$ or $\mathbb{F}\left[\mathfrak{g}\langle\sigma\rangle^{*}\right]^{G\langle\sigma\rangle}$ [17, Prop. 3.1]. In the context of quaternionic decompositions, we may extend the scope of this method beyond the (co)adjoint representations.

Recall that we work with a quaternionic decomposition 


$$
\mathfrak{g}=\begin{array}{c:c}
\mathfrak{g}_{00} & \mathfrak{g}_{01} \\
\hdashline \mathfrak{g}_{10} & \mathfrak{g}_{11}
\end{array} \sigma_{1}
$$

and $\sigma_{3}=\sigma_{1} \sigma_{2}$. Consider the $\mathbb{Z}_{2}$-contraction of $\mathfrak{g}^{\sigma_{1}}$ with respect to $\sigma_{2}$ (or $\sigma_{3}$, which is the same), that is, set $\mathfrak{k}_{01}:=\mathfrak{g}^{\sigma_{1}}\left\langle\sigma_{2}\right\rangle=\mathfrak{g}_{00} \ltimes \mathfrak{g}_{01}^{a}$. The vector space $\mathfrak{g}_{1 \star}$ can be regarded as $\mathfrak{k}_{01}$-module in two different ways. In both cases, the subalgebra $\mathfrak{g}_{00} \subset \mathfrak{k}_{01}$ acts on $\mathfrak{g}_{1 \star}$ as it was in $\mathfrak{g}$. Let $x \in \mathfrak{g}_{01}$ and $\left(y_{0}, y_{1}\right) \in \mathfrak{g}_{10} \oplus \mathfrak{g}_{11}$. For the action of the abelian nilpotent radical $\mathfrak{g}_{01}^{a} \subset \mathfrak{k}_{01}$, the two possibilities are:

a) $x \cdot\left(y_{0}, y_{1}\right)=\left(0,\left[x, y_{0}\right]\right)$;

b) $x \cdot\left(y_{0}, y_{1}\right)=\left(\left[x, y_{1}\right], 0\right)$.

The $\mathfrak{k}_{01}$-modules obtained in this way are denoted by $\mathfrak{g}_{10} \propto \mathfrak{g}_{11}$ and $\mathfrak{g}_{11} \propto \mathfrak{g}_{10}$, respectively. From a slightly different angle, these possibilities can be realised as follows:

$\left.\mathrm{a}^{\prime}\right)$ Take the $\mathbb{Z}_{2}$-contraction of $\mathfrak{g}$ with respect to $\sigma_{2}$, i.e., $\mathfrak{g}\left\langle\sigma_{2}\right\rangle=\mathfrak{g}_{\star 0} \ltimes \mathfrak{g}_{\star 1}^{a}$. Identifying $\mathfrak{g}$ and $\mathfrak{g}\left\langle\sigma_{2}\right\rangle$ as vector spaces, we notice that the linear operators $\sigma_{1}$ and $\sigma_{3}$ remain involutions of the Lie algebra $\mathfrak{g}\left\langle\sigma_{2}\right\rangle$. Taking the eigenspaces of $\sigma_{1}$ in $\mathfrak{g}\left\langle\sigma_{2}\right\rangle$ yields $\mathfrak{g}\left\langle\sigma_{2}\right\rangle^{\sigma_{1}}=\mathfrak{k}_{01}$ and $\mathfrak{g}\left\langle\sigma_{2}\right\rangle_{1}^{\left(\sigma_{1}\right)}=\mathfrak{g}_{10} \propto \mathfrak{g}_{11}$.

$\left.\mathrm{b}^{\prime}\right)$ Likewise, starting with $\mathfrak{g}\left\langle\sigma_{3}\right\rangle$, we end up with $\mathfrak{g}\left\langle\sigma_{3}\right\rangle^{\sigma_{1}}=\mathfrak{k}_{01}$ and $\mathfrak{g}\left\langle\sigma_{3}\right\rangle_{1}^{\left(\sigma_{1}\right)}=\mathfrak{g}_{11} \propto \mathfrak{g}_{10}$.

We have $G\left\langle\sigma_{2}\right\rangle=G_{\star 0} \ltimes \exp \left(\mathfrak{g}_{\star 1}^{a}\right)$ and both $G_{\star 0}$ and $\mathfrak{g}_{\star 1}$ are $\sigma_{1}$-stable. It follows that $\sigma_{1}$ can be lifted to an involution of $G\left\langle\sigma_{2}\right\rangle$ and the identity component of $G\left\langle\sigma_{2}\right\rangle^{\sigma_{1}}$ is $K_{01}=$ $G_{00} \ltimes \exp \left(\mathfrak{g}_{01}^{a}\right)=: G_{00} \ltimes N_{01}$. (Likewise, $K_{01}$ is the identity component of $G\left\langle\sigma_{3}\right\rangle^{\sigma_{1}}$.) The exponential map exp : $\mathfrak{g}_{01} \rightarrow N_{01}$ is an isomorphism of varieties and the action of $N_{01}$ is given by

$$
\begin{aligned}
& \exp (x) \cdot\left(y_{0}, y_{1}\right)=\left(y_{0}, y_{1}\right)+x \cdot\left(y_{0}, y_{1}\right)=\left(y_{0}, y_{1}+\left[x, y_{0}\right]\right) \text { for }\left(y_{0}, y_{1}\right) \in \mathfrak{g}_{10} \propto \mathfrak{g}_{11}, \\
& \exp (x) \cdot\left(y_{1}, y_{0}\right)=\left(y_{1}, y_{0}\right)+x \cdot\left(y_{1}, y_{0}\right)=\left(y_{1}, y_{0}+\left[x, y_{1}\right]\right) \text { for }\left(y_{1}, y_{0}\right) \in \mathfrak{g}_{11} \propto \mathfrak{g}_{10} .
\end{aligned}
$$

Note that $G_{00}$ is a subgroup of both $K_{01}$ and $G_{0 \star}$, and the action of $G_{00}$ does not vary under the passage from the $G_{0 \star}$-module $\mathfrak{g}_{1 \star}$ to the $K_{01}$-modules $\mathfrak{g}_{10} \propto \mathfrak{g}_{11}$ or $\mathfrak{g}_{11} \propto \mathfrak{g}_{10}$. Summarising the previous discussion, we get the following:

Claim 4.1. 1) The involution $\sigma_{1} \in \operatorname{Inv}(\mathfrak{g})$ can be regarded as involution of $\mathfrak{g}\left\langle\sigma_{2}\right\rangle$ and of $G\left\langle\sigma_{2}\right\rangle$. The group $K_{01}$ is the identity component of $G\left\langle\sigma_{2}\right\rangle^{\sigma_{1}}$ and the corresponding isotropy representation is $\left(K_{01}: \mathfrak{g}_{10} \propto \mathfrak{g}_{11}\right)$.

2) Likewise, $K_{01}$ is the identity component of $G\left\langle\sigma_{3}\right\rangle{ }^{\sigma_{1}}$ and the corresponding isotropy representation is $\left(K_{01}: \mathfrak{g}_{11} \propto \mathfrak{g}_{10}\right)$.

3) Both representations can be understood as different degenerations of $\left(G_{0 \star}: \mathfrak{g}_{1 \star}\right)$. The symmetric spaces $G\left\langle\sigma_{2}\right\rangle / K_{01}$ and $G\left\langle\sigma_{3}\right\rangle / K_{01}$ can be regarded as different degenerations of $G / G_{\star 0}$.

Lemma 4.2. The $K_{01}$-modules $\mathfrak{g}_{10} \propto \mathfrak{g}_{11}$ and $\mathfrak{g}_{11} \propto \mathfrak{g}_{10}$ are dual to each other. 
Proof. If $\bar{y}=\left(y_{0}, y_{1}\right) \in \mathfrak{g}_{10} \propto \mathfrak{g}_{11}$ and $\bar{z}=\left(z_{1}, z_{0}\right) \in \mathfrak{g}_{11} \propto \mathfrak{g}_{10}$, then $(\bar{y}, \bar{z}) \mapsto \kappa\left(y_{0}, z_{0}\right)+\kappa\left(y_{1}, z_{1}\right)$ is the required $K_{01}$-invariant pairing.

Lemma 4.3. The homogeneous space $G\left\langle\sigma_{2}\right\rangle / K_{01}$ is affine.

Proof. Let $\mathcal{A}^{u}$ denote the unipotent radical of an algebraic group $\mathcal{A}$. By construction, $G\left\langle\sigma_{2}\right\rangle^{u}=\exp \left(\mathfrak{g}_{\star 1}^{a}\right)$ and $\left(K_{01}\right)^{u}=\exp \left(\mathfrak{g}_{01}^{a}\right)$. Hence $\left(K_{01}\right)^{u} \subset G\left\langle\sigma_{2}\right\rangle^{u}$. By [2, Cor. 2], this condition is sufficient for $G\left\langle\sigma_{2}\right\rangle / K_{01}$ to be affine.

Hint. Using the notation $\mathfrak{g}_{10} \propto \mathfrak{g}_{11}$ or $\mathfrak{g}_{11} \propto \mathfrak{g}_{10}$ always means that the vector space $\mathfrak{g}_{1 \star}$ is regarded as a $K_{01}$-module in the prescribed way.

Similar notation is used for the other $\mathbb{Z}_{2}$-contractions of $\mathfrak{g}$ and their involutions. For instance, $\mathfrak{g}\left\langle\sigma_{1}\right\rangle^{\sigma_{2}}=\mathfrak{g}\left\langle\sigma_{3}\right\rangle^{\sigma_{2}}=\mathfrak{g}_{00} \ltimes \mathfrak{g}_{10}^{a}=: \mathfrak{k}_{10}, \mathfrak{g}\left\langle\sigma_{1}\right\rangle_{1}^{\left(\sigma_{2}\right)}=\mathfrak{g}_{01} \propto \mathfrak{g}_{11}$, and $\mathfrak{g}\left\langle\sigma_{3}\right\rangle_{1}^{\left(\sigma_{2}\right)}=\mathfrak{g}_{11} \propto \mathfrak{g}_{01}$. Then $N_{10}=\exp \left(\mathfrak{g}_{10}^{a}\right)$ and $K_{10}=G_{00} \ltimes N_{10}$ acts on $\mathfrak{g}_{01} \propto \mathfrak{g}_{11}$ or $\mathfrak{g}_{11} \propto \mathfrak{g}_{01}$. In this way, we obtain 6 new isotropy representations related to 3 possible $\mathbb{Z}_{2}$-contractions of $\mathfrak{g}$.

Example 4.4. The adjoint and coadjoint representations of any $\mathbb{Z}_{2}$-contraction can be obtained as a special case of this construction. Given $\sigma \in \operatorname{lnv}(\mathfrak{g})$, consider three involutions of $\tilde{\mathfrak{g}}=\mathfrak{g} \oplus \mathfrak{g}$ as in Example 3.10:

$$
\sigma_{1}(x, y)=(y, x), \sigma_{2}(x, y)=(\sigma(x), \sigma(y)), \text { and } \sigma_{3}=\sigma_{1} \sigma_{2} .
$$

Then $\tilde{\mathfrak{g}}\left\langle\sigma_{2}\right\rangle^{\sigma_{1}}=\mathfrak{g}\langle\sigma\rangle$ and $\tilde{\mathfrak{g}}\left\langle\sigma_{2}\right\rangle_{1}^{\left(\sigma_{1}\right)}$ is isomorphic to the adjoint module of $\mathfrak{g}\langle\sigma\rangle$; whereas $\tilde{\mathfrak{g}}\left\langle\sigma_{3}\right\rangle^{\sigma_{1}}=\mathfrak{g}\langle\sigma\rangle$ and $\tilde{\mathfrak{g}}\left\langle\sigma_{3}\right\rangle_{1}^{\left(\sigma_{1}\right)}$ is isomorphic to the coadjoint module of $\mathfrak{g}\langle\sigma\rangle$.

Convention. Whenever it is notationally convenient, we will expose our results in the most symmetric form, i.e., for an arbitrary permutation of indices $(10,01,11)$. Otherwise, we stick to our sample choice with $K=K_{01}$ and the $K$-module $V=\mathfrak{g}_{10} \propto \mathfrak{g}_{11}$.

Let $(\alpha, \beta, \gamma)$ be a permutation of $(10,01,11)$. The representation of $K_{\beta}=G_{00} \ltimes N_{\beta}$ in $V_{\alpha \gamma}:=\mathfrak{g}_{\alpha} \propto \mathfrak{g}_{\gamma}$ is said to be a degenerated isotropy representation. There is a special situation in which the algebra $\mathbb{F}\left[V_{\alpha \gamma}\right]^{K_{\beta}}$ can explicitly be described. Since $\mathbb{F}\left[V_{\alpha \gamma}\right]^{K_{\beta}}=\left(\mathbb{F}\left[V_{\alpha \gamma}\right]^{N_{\beta}}\right)^{G_{00}}$, the first step is to describe the algebra $\mathbb{F}\left[V_{\alpha \gamma}\right]^{N_{\beta}}$. It follows from the appropriate analogue of (4.1) that the dimension of any $N_{\beta}$-orbit in $V_{\alpha \gamma}$ is at most $\operatorname{dim} \mathfrak{g}_{\gamma}$ and if $p: V_{\alpha \gamma} \rightarrow \mathfrak{g}_{\alpha}$ is the projection along $\mathfrak{g}_{\gamma}$, then $\mathbb{F}\left[\mathfrak{g}_{\alpha}\right] \simeq p^{*}\left(\mathbb{F}\left[\mathfrak{g}_{\alpha}\right]\right) \subset \mathbb{F}\left[V_{\alpha \gamma}\right]^{N_{\beta}}$.

Theorem 4.5. Suppose that there is $\tilde{y} \in \mathfrak{g}_{\alpha}$ such that $\left[\tilde{y}, \mathfrak{g}_{\beta}\right]=\mathfrak{g}_{\gamma}$. Then

(i) $\mathbb{F}\left[V_{\alpha \gamma}\right]^{N_{\beta}} \simeq \mathbb{F}\left[\mathfrak{g}_{\alpha}\right]$,

(ii) $\mathbb{F}\left[V_{\alpha \gamma}\right]^{K_{\beta}} \simeq \mathbb{F}\left[\mathfrak{g}_{\alpha}\right]^{G_{00}}$ is a polynomial algebra,

(iii) $\mathbb{F}\left[V_{\alpha \gamma}\right]$ is a free $\mathbb{F}\left[V_{\alpha \gamma}\right]^{K_{\beta}}$-module.

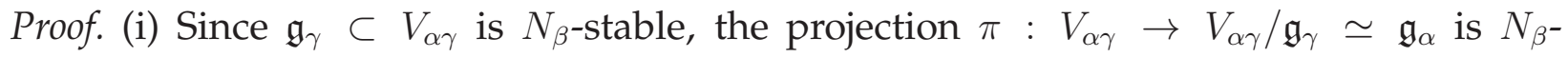
equivariant. Moreover, the induced $N_{\beta}$-action on $V_{\alpha \gamma} / \mathfrak{g}_{\gamma}$ is trivial. In order to prove that $\pi$ is the quotient by $N_{\beta}$, we use the Igusa lemma, see e.g. [16, Lemma 6.1]. Since $\pi$ is 
onto and $V_{\alpha \gamma} / \mathfrak{g}_{\gamma}$ is normal, it suffices to prove that generic fibres of $\pi$ are $N_{\beta}$-orbits. If $\left[\tilde{y}, \mathfrak{g}_{\beta}\right]=\mathfrak{g}_{\gamma}$ and we identify $\tilde{y}$ with $(\tilde{y}, 0) \in V_{\alpha \gamma}$, then the orbit $N_{\beta} \cdot \tilde{y}=\tilde{y}+\left[\tilde{y}, \mathfrak{g}_{\beta}\right]$ is precisely a fibre of $\pi$. The appropriate analogue of Eq. (4.1) shows that $\operatorname{dim} N_{\beta} \cdot\left(y^{\prime}, y^{\prime \prime}\right)=\operatorname{dim}\left[y^{\prime}, \mathfrak{g}_{\beta}\right]$; in particular, it depends only on $y^{\prime} \in \mathfrak{g}_{\alpha}$. Consequently, there is a dense open subset $\Omega \subset \mathfrak{g}_{\alpha}$ such that $\left[y, \mathfrak{g}_{\beta}\right]=\mathfrak{g}_{\gamma}$ for all $y \in \Omega$. Finally, identifying $\Omega$ with an open subset in $V_{\alpha \gamma} / \mathfrak{g}_{\gamma}$, we see that $\pi^{-1}(y)$ is a sole orbit for all $y \in \Omega$.

(ii), (iii) This follows from (i) and the corresponding properties of the algebra of invariants for the isotropy representation $\left(G_{00}: \mathfrak{g}_{\alpha}\right)$.

The above proof shows that $\mathbb{F}\left[V_{\alpha \gamma}\right]^{N_{\beta}}=p^{*}\left(\mathbb{F}\left[\mathfrak{g}_{\alpha}\right]\right)$ if and only if there is $\tilde{y} \in \mathfrak{g}_{\alpha}$ such that $\left[\tilde{y}, \mathfrak{g}_{\beta}\right]=\mathfrak{g}_{\gamma}$ if and only if $\max _{v \in V_{\alpha \gamma}} \operatorname{dim} N_{\beta} \cdot v=\operatorname{dim} \mathfrak{g}_{\gamma}$.

We will see in a moment that the hypothesis of Theorem 4.5 is equivalent to a coincidence of CSS.

Lemma 4.6. For any quaternionic decomposition and $x \in \mathfrak{g}_{\alpha}$, we have $\operatorname{dim}\left[\mathfrak{g}_{\beta}, x\right]=\operatorname{dim}\left[\mathfrak{g}_{\gamma}, x\right]$.

Proof. Set $d_{i j}=\operatorname{dim} \mathfrak{z}_{\mathfrak{g}}(x)_{i j}$. Applying [11, Prop. 5] to one little and one big $\mathbb{Z}_{2}$-grading, we obtain:

$$
\begin{aligned}
d_{\alpha}-d_{00} & = & \operatorname{dim} \mathfrak{g}_{\alpha} & -\operatorname{dim} \mathfrak{g}_{00} \\
\left(d_{\alpha}+d_{\gamma}\right)-\left(d_{00}+d_{\beta}\right) & = & \operatorname{dim}\left(\mathfrak{g}_{\alpha} \oplus \mathfrak{g}_{\gamma}\right) & -\operatorname{dim}\left(\mathfrak{g}_{00} \oplus \mathfrak{g}_{\beta}\right) .
\end{aligned}
$$

Taking the difference yields $d_{\gamma}-d_{\beta}=\operatorname{dim} \mathfrak{g}_{\gamma}-\operatorname{dim} \mathfrak{g}_{\beta}$, as required.

Proposition 4.7. There exists $x \in \mathfrak{g}_{\alpha}$ such that $\left[\mathfrak{g}_{\beta}, x\right]=\mathfrak{g}_{\gamma}$ if and only if any CSS $\mathfrak{c}_{\alpha} \subset \mathfrak{g}_{\alpha}$ is also a CSS in $\mathfrak{g}_{\alpha} \oplus \mathfrak{g}_{\gamma}$;

Proof. " $\Rightarrow$ ". The set of elements $x \in \mathfrak{g}_{\alpha}$ having such property is open. If $x$ is $G_{00}$-regular and semisimple, then $\mathfrak{z}_{\mathfrak{g}}(x)_{\alpha}$ is a CSS in $\mathfrak{g}_{\alpha}$. By Lemma 4.6, the assumption implies that $\mathfrak{z}_{\mathfrak{g}}(x)_{\gamma}=\{0\}$. Thus, the CSS $\mathfrak{c}_{\alpha}:=\mathfrak{z}_{\mathfrak{g}}(x)_{\alpha}$ has the property that $\mathfrak{z}_{\mathfrak{g}}\left(\mathfrak{c}_{\alpha}\right) \cap\left(\mathfrak{g}_{\alpha} \oplus \mathfrak{g}_{\gamma}\right)=\mathfrak{z}_{\mathfrak{g}}(x) \cap$ $\left(\mathfrak{g}_{\alpha} \oplus \mathfrak{g}_{\gamma}\right)=\mathfrak{c}_{\alpha}$, i.e., $\mathfrak{c}_{\alpha}$ is a CSS in $\mathfrak{g}_{\alpha} \oplus \mathfrak{g}_{\gamma}$.

" $\Leftarrow "$. Reversing the preceding argument shows that any $G_{00}$-regular semsimple element $x \in \mathfrak{c}_{\alpha}$ has the required property.

This proposition yields a simple necessary condition for coincidence:

Corollary 4.8. If $\mathfrak{c}_{\alpha}$ is also a CSS in $\mathfrak{g}_{\alpha} \oplus \mathfrak{g}_{\gamma}$, then $\operatorname{dim} \mathfrak{g}_{\beta} \geqslant \operatorname{dim} \mathfrak{g}_{\gamma}$.

Combining our previous results, we get an explicit description of the algebra of invariants for certain degenerated isotropy representations.

Theorem 4.9. Let $\mathfrak{g}$ be a semisimple Lie algebra and $\left\{\sigma_{1}, \sigma_{2}, \sigma_{3}\right\}$ a triple of involutions of $\mathfrak{g}$ such that $\sigma_{1} \sigma_{2}=\sigma_{3}$.

(i) Suppose that $\sigma_{1}$ is of maximal rank. Then

$$
\mathbb{F}\left[\mathfrak{g}_{11} \propto \mathfrak{g}_{01}\right]^{G_{00} \ltimes N_{10}} \simeq \mathbb{F}\left[\mathfrak{g}_{11}\right]^{G_{00}} \text { and } \mathbb{F}\left[\mathfrak{g}_{10} \propto \mathfrak{g}_{01}\right]^{G_{00} \ltimes N_{11}} \simeq \mathbb{F}\left[\mathfrak{g}_{10}\right]^{G_{00}} \text {. }
$$


(ii) Suppose that $\left\{\sigma_{1}, \sigma_{2}\right\}$ is a dyad. Then

$\mathbb{F}\left[\mathfrak{g}_{11} \propto \mathfrak{g}_{10}\right]^{G_{00} \ltimes N_{01}} \simeq \mathbb{F}\left[\mathfrak{g}_{11}\right]^{G_{00}}$ and $\mathbb{F}\left[\mathfrak{g}_{11} \propto \mathfrak{g}_{01}\right]^{G_{00} \ltimes N_{10}} \simeq \mathbb{F}\left[\mathfrak{g}_{11}\right]^{G_{00}}$.

(iii) Suppose that $\left\{\sigma_{1}, \sigma_{2}, \sigma_{3}\right\}$ is a triad, then $\mathbb{F}\left[\mathfrak{g}_{\alpha} \ltimes \mathfrak{g}_{\gamma}\right]^{G_{00} \ltimes N_{\beta}} \simeq \mathbb{F}\left[\mathfrak{g}_{\alpha}\right]^{G_{00}}$ for all permutations $(\alpha, \beta, \gamma)$ of $(01,10,11)$.

In all previous cases, $\mathbb{F}\left[\mathfrak{g}_{\alpha} \ltimes \mathfrak{g}_{\gamma}\right]^{N_{\beta}}=\mathbb{F}\left[\mathfrak{g}_{\alpha}\right]$ and $\mathbb{F}\left[\mathfrak{g}_{\alpha} \ltimes \mathfrak{g}_{\gamma}\right]$ is a free $\mathbb{F}\left[\mathfrak{g}_{\alpha} \ltimes \mathfrak{g}_{\gamma}\right]^{G_{00} \ltimes N_{\beta} \text {-module. }}$

Proof. (i) Combine Theorem 3.3, Theorem 4.5, and Proposition 4.7.

(ii) Combine Theorem 3.7, Theorem 4.5, and Proposition 4.7.

(iii) Combine Corollary 3.8, Theorem 4.5, and Proposition 4.7.

If $\left[\mathfrak{g}_{\beta}, x\right]$ is a proper subspace of $\mathfrak{g}_{\gamma}$ for all $x \in \mathfrak{g}_{\alpha}$, i.e., the maximal dimension of $N_{\beta^{-}}$ orbits in $V_{\alpha \gamma}$ is less than $\operatorname{dim} \mathfrak{g}_{\gamma}$, then a general description of $\mathbb{F}\left[V_{\alpha \gamma}\right]^{N_{\beta}}$ (and hence of $\left.\mathbb{F}\left[V_{\alpha \gamma}\right]^{K_{\beta}}\right)$ is not available. Nevertheless, the $K_{\beta}$-module $V_{\alpha \gamma}$ always retains some good properties of initial isotropy representation of a reductive group.

Below, we consider the problem of existence of a generic stabiliser for the linear action of an algebraic group $K$ on $V$. Recall that a subalgebra $\mathfrak{q} \subset \mathfrak{k}$ is a generic stabiliser if there exists a dense open subset $\Omega \subset V$ such that the stabiliser $\mathfrak{k}^{v}=\{k \in \mathfrak{k} \mid k \cdot v=0\}$ is $K$ conjugate to $\mathfrak{q}$ for all $v \in \Omega$. We then write $\mathfrak{q}=$ g.s. $(K: V)$ and the elements of $\Omega$ are called $K$-generic points (or just generic points if the group is clear from the context). A generic stabiliser always exists for the reductive group actions on smooth affine varieties, see e.g. $[27, \S 7]$. Moreover, for the isotropy representation $\left(G_{0}: \mathfrak{g}_{1}\right), x \in \mathfrak{g}_{1}$ is $G_{0}$-generic if and only if $x$ is $G_{0}$-regular and semisimple if and only if $\mathfrak{z}_{\mathfrak{g}}(x) \cap \mathfrak{g}_{1}$ is a CSS in $\mathfrak{g}_{1}$.

In the following theorem, we write $\mathfrak{g}^{\xi}$ in place of $\mathfrak{z}_{\mathfrak{g}}(\xi)(\xi \in \mathfrak{g})$, and $\mathfrak{g}_{i j}^{\xi}:=\mathfrak{g}^{\xi} \cap \mathfrak{g}_{i j}$.

Theorem 4.10. (i) The degenerated isotropy representation $\left(K=G_{00} \ltimes N_{01}: V=\mathfrak{g}_{10} \propto \mathfrak{g}_{11}\right)$ always has a generic stabiliser. More precisely, let $\xi \in \mathfrak{g}_{10}$ be a $G_{00}$-generic point for the (little isotropy) representation $\left(G_{00}: \mathfrak{g}_{10}\right)$ and let $\eta$ be a generic point in the $G_{00}^{\xi}$-module $\mathfrak{g}_{11}^{\xi}$. Then $v=(\xi, \eta)$ is a K-generic point in $V$ and g.s. $(K: V)=$ g.s. $\left(G_{00}^{\xi}: \mathfrak{g}_{11}^{\xi}\right) \ltimes\left(\mathfrak{g}_{01}^{\xi}\right)^{a}$.

(ii) $\operatorname{trdeg} \mathbb{F}(V)^{K}=\operatorname{dim} \mathfrak{g}_{10} / / G_{00}+\operatorname{dim} \mathfrak{g}_{11}^{\xi} / / G_{00}^{\xi}$.

(iii) $\mathbb{F}(V)^{K}$ is the fraction field of $\mathbb{F}[V]^{K}$.

Proof. (i) As explained above, $\xi$ is semisimple and $\mathfrak{c}_{10}:=\mathfrak{g}_{10}^{\xi}$ is a CSS in $\mathfrak{g}_{10}$. Then

$$
\mathfrak{g}_{11}=\left[\mathfrak{g}_{01}, \xi\right] \oplus \mathfrak{g}_{11}^{\xi} .
$$

Note that $\mathfrak{g}_{00}^{\xi} \oplus \mathfrak{g}_{11}^{\xi}$ is a $\mathbb{Z}_{2}$-grading of the reductive Lie algebra $\mathfrak{g}^{\sigma_{3}} \cap \mathfrak{g}^{\xi}$. Let $\tilde{\mathfrak{c}}$ be a CSS in $\mathfrak{g}_{11}^{\xi}$ and let $\eta \in \tilde{\mathfrak{c}}$ be a $G_{00}^{\xi}$-generic point. The stabiliser of $(\xi, \eta)$ in $\mathfrak{k}$ is determined by the following conditions:

$$
\mathfrak{k}^{(\xi, \eta)}=\left\{\left(s_{0}, s_{1}\right) \in \mathfrak{g}_{00} \ltimes \mathfrak{g}_{01}^{a} \mid\left[s_{0}, \xi\right]=0 \&\left[s_{0}, \eta\right]+\left[s_{1}, \xi\right]=0\right\} .
$$


Hence $s_{0} \in \mathfrak{g}_{00}^{\xi}$. Then the second equation and (4.2) show that $\left[s_{0}, \eta\right]=0$ and $\left[s_{1}, \xi\right]=0$; that is, $s_{0} \in\left(\mathfrak{g}_{00}^{\xi}\right)^{\eta}$ and $s_{1} \in \mathfrak{g}_{01}^{\xi}$. Thus,

$$
\mathfrak{k}^{(\xi, \eta)}=\left(\mathfrak{g}_{00}^{\xi}\right)^{\eta} \ltimes\left(\mathfrak{g}_{01}^{\xi}\right)^{a}=\text { g.s. }\left(G_{00}^{\xi}: \mathfrak{g}_{11}^{\xi}\right) \ltimes\left(\mathfrak{g}_{01}^{\xi}\right)^{a} .
$$

Note that this stabiliser does not essentially depend on $(\xi, \eta)$. If $\xi^{\prime} \in \mathfrak{c}_{10}$ and $\eta^{\prime} \in \tilde{\mathfrak{c}}$ are some other generic points, then $\mathfrak{k}^{(\xi, \eta)}=\mathfrak{k}^{\left(\xi^{\prime}, \eta^{\prime}\right)}$. In view of this observation, to prove that $\mathfrak{k}^{(\xi, \eta)}$ is a generic stabiliser, it is sufficient to show that the $K$-saturation of $\mathfrak{c}_{10} \propto \tilde{\mathfrak{c}} \subset \mathfrak{g}_{10} \propto \mathfrak{g}_{11}$ is dense.

By our constructions, there are the following relations for $\mathfrak{c}_{10}$ and $\tilde{\mathfrak{c}}$ :

- $G_{00}^{\xi} \cdot \tilde{\mathfrak{c}}$ is dense in $\mathfrak{g}_{11}^{\xi}$;

- $\quad N_{01} \cdot\left(\xi^{\prime}, \eta^{\prime}\right)=\left(\xi^{\prime},\left[\mathfrak{g}_{01}, \xi^{\prime}\right]+\eta^{\prime}\right)$ and $\left[\mathfrak{g}_{01}, \xi^{\prime}\right]=\left[\mathfrak{g}_{01}, \xi\right]$ if $\xi^{\prime} \in \mathfrak{c}_{10}$ is generic;

- $G_{00} \cdot \mathfrak{c}_{10}$ is dense in $\mathfrak{g}_{10}$.

The first two relations and the fact that $G_{00}^{\xi}$ does not affect $\mathfrak{c}_{10}=\mathfrak{g}_{11}^{\xi}$ show us that $\left(G_{00}^{\xi} \ltimes\right.$ $\left.N_{01}\right) \cdot\left(\mathfrak{c}_{10} \propto \mathfrak{c}\right)$ is dense in $\mathfrak{c}_{10} \propto \mathfrak{g}_{11}$. Then the last relation implies that $G_{00} \cdot\left(\mathfrak{c}_{10} \propto \mathfrak{g}_{11}\right)$ is dense in $V$.

(ii) By (i) and the Rosenlicht theorem [27, 2.3], we have

$$
\begin{aligned}
& \operatorname{dim} \text { g.s. }(K: V)=\operatorname{dim} \mathfrak{g}_{01}^{\xi}+\operatorname{dim} \text { g.s. }\left(G_{00}^{\xi}: \mathfrak{g}_{11}^{\xi}\right)= \\
& \qquad \operatorname{dim} \mathfrak{g}_{01}^{\xi}+\operatorname{dim} \mathfrak{g}_{00}^{\xi}-\operatorname{dim} \mathfrak{g}_{11}^{\xi}+\operatorname{trdeg} \mathbb{F}\left(\mathfrak{g}_{11}^{\xi}\right)^{G_{00}^{\xi}} .
\end{aligned}
$$

Since $\mathfrak{g}_{11}^{\xi}$ is an orthogonal $G_{00}^{\xi}$-module, it follows from [12] that the field $\mathbb{F}\left(\mathfrak{g}_{11}^{\xi}\right)^{G_{00}^{\xi}}$ is the fraction field of the algebra $\mathbb{F}\left[\mathfrak{g}_{11}^{\xi}\right]^{G_{00}^{\xi}}$. Hence $\operatorname{trdeg} \mathbb{F}\left(\mathfrak{g}_{11}^{\xi}\right)^{G_{00}^{\xi}}=\operatorname{dim} \mathfrak{g}_{11}^{\xi} / / G_{00}^{\xi}$. Then using again the Rosenlicht theorem, we obtain

$$
\begin{aligned}
& \operatorname{trdeg} \mathbb{F}(V)^{K} \stackrel{\text { Rosenlicht }}{=} \operatorname{dim} V-\operatorname{dim} K+\operatorname{dim} \text { g.s. }(K: V)= \\
& \left.\underline{\left(\operatorname{dim} \mathfrak{g}_{11}\right.}+\operatorname{dim} \mathfrak{g}_{10}\right)-\left(\underline{\operatorname{dim} \mathfrak{g}_{01}}+\operatorname{dim} \mathfrak{g}_{00}\right)+\left(\underline{\operatorname{dim} \mathfrak{g}_{01}^{\xi}}+\operatorname{dim} \mathfrak{g}_{00}^{\xi}-\underline{\operatorname{dim} \mathfrak{g}_{11}^{\xi}}+\operatorname{dim} \mathfrak{g}_{11}^{\xi} / / G_{00}^{\xi}\right)= \\
& \left(\operatorname{dim} \mathfrak{g}_{10}-\operatorname{dim} \mathfrak{g}_{00}+\operatorname{dim} \mathfrak{g}_{00}^{\xi}\right)+\operatorname{dim} \mathfrak{g}_{11}^{\xi} / / G_{00}^{\xi}=\operatorname{dim} \mathfrak{g}_{10} / / G_{00}+\operatorname{dim} \mathfrak{g}_{11}^{\xi} / / G_{00}^{\xi},
\end{aligned}
$$

where the underlined terms in the second line are cancelled out, in view of Lemma 4.6 applied to $\xi \in \mathfrak{g}_{10}$.

(iii) The proof of [17, Lemma 2.6] applies in this situation.

Corollary 4.11. The following conditions are equivalent: (i) $\operatorname{trdeg} \mathbb{F}(V)^{K}=\operatorname{dim} \mathfrak{g}_{10} / / G_{00}$, (ii) $\mathfrak{g}_{11}^{\xi}=0$ for almost all $\xi \in \mathfrak{g}_{10}$, (iii) any CSS $\mathfrak{c}_{10} \subset \mathfrak{g}_{10}$ is also a CSS in $\mathfrak{g}_{10} \oplus \mathfrak{g}_{11}$, (iv) $\mathbb{F}[V]^{K} \simeq$ $\mathbb{F}\left[\mathfrak{g}_{10}\right]^{G 00}$.

Corollary 4.12. It is always true that $\operatorname{trdeg} \mathbb{F}(V)^{K}=\operatorname{dim} \mathfrak{g}_{1 \star} / / G_{0 \star}=\operatorname{trdeg} \mathbb{F}\left(\mathfrak{g}_{1 \star}\right)^{G_{0 \star}}$.

Proof. Choose a CSS $\mathfrak{c}_{10} \subset \mathfrak{g}_{10}$. By [11], $\operatorname{dim} \mathfrak{g}_{10} / / G_{00}=\operatorname{dim} \mathfrak{c}_{10}$ and $\operatorname{dim} \mathfrak{g}_{11}^{\xi} / / G_{00}^{\xi}$ is the dimension of any CSS in $\mathfrak{g}_{11}^{\xi}$. Without loss of generality, we may assume that $\xi \in \mathfrak{c}_{10}$ and $\mathfrak{z}_{\mathfrak{g}}(\xi)=\mathfrak{z}_{\mathfrak{g}}\left(\mathfrak{c}_{10}\right)$. Therefore, if $\mathfrak{h}$ is a Cartan subspace in $\mathfrak{g}_{11}^{\xi}=\mathfrak{z}_{\mathfrak{g}}\left(\mathfrak{c}_{10}\right)_{11}$, then $\mathfrak{c}_{10} \oplus \mathfrak{h}$ is a CSS 
in $\mathfrak{g}_{1 \star}$. That is, the right-hand side in Theorem 4.10 (ii) is exactly $\operatorname{dim} \mathfrak{g}_{1 \star} / / G_{0 \star}$. The second equality is a general property of orthogonal representations of reductive groups [12].

There is a general 'contraction procedure' for obtaining $K$-invariants in $\mathbb{F}[V]$ or $\mathbb{F}\left[V^{*}\right]$. Recall that $V$ and $V^{*}$ coincide as vector spaces and are isomorphic as $G_{00}$-modules. Only the $N_{01}$-actions on them are different. Therefore, $\mathbb{F}[V]^{K}$ and $\mathbb{F}\left[V^{*}\right]^{K}$ can be regarded as subalgebras of $\mathbb{F}\left[\mathfrak{g}_{10} \oplus \mathfrak{g}_{11}\right]^{G_{00}}$. Let $\mathbb{F}\left[\mathfrak{g}_{10} \oplus \mathfrak{g}_{11}\right]_{(a, b)}$ denote the space of bi-homogeneous polynomials of degree $a$ with respect to $\mathfrak{g}_{10}$ and degree $b$ with respect to $\mathfrak{g}_{11}$. Any homogeneous polynomial $f \in \mathbb{F}\left[\mathfrak{g}_{10} \oplus \mathfrak{g}_{11}\right]$ of degree $n$ can be decomposed into the sum of bi-homogeneous components $f=\sum_{i=k}^{m} f_{i}$, where $f_{i} \in \mathbb{F}\left[\mathfrak{g}_{10} \oplus \mathfrak{g}_{11}\right]_{(n-i, i)}$. Assuming that $f_{k}, f_{m} \neq 0$, we set $f^{\bullet}:=f_{k}$ and $f_{\bullet}:=f_{m}$. That is, $f^{\bullet}$ is the bi-homogeneous component having the maximal degree w.r.t. $\mathfrak{g}_{10}$.

Proposition 4.13. Suppose that $f \in \mathbb{F}\left[\mathfrak{g}_{1 \star}\right]^{G_{0 \star}}$ is homogeneous. Then

$$
f^{\bullet} \in \mathbb{F}\left[\mathfrak{g}_{10} \propto \mathfrak{g}_{11}\right]^{K} \text { and } f_{\bullet} \in \mathbb{F}\left[\mathfrak{g}_{11} \propto \mathfrak{g}_{10}\right]^{K} .
$$

Proof. The proof is similar to that of [17, Prop.3.1], which was designed for the adjoint and coadjoint representations of $K$. For convenience, we repeat it here. Clearly, each bi-homogeneous component $f_{i}$ of $f$ is $G_{00}$-invariant. For $x \in \mathfrak{g}_{01}$, let $\mathcal{D}_{x}$ denote the corresponding derivation of $\mathbb{F}\left[\mathfrak{g}_{1 \star}\right]$. (Here we regard the space $\mathfrak{g}_{1 \star}$ as $G_{0 \star}$-module, i.e., $x$ is an element of the Lie algebra $\left.\mathfrak{g}_{0 \star}.\right)$ Since ad $x\left(\mathfrak{g}_{10}\right) \subset \mathfrak{g}_{11}$ and $\operatorname{ad} x\left(\mathfrak{g}_{11}\right) \subset \mathfrak{g}_{10}$, we have

$$
\mathcal{D}_{x}\left(\mathbb{F}\left[\mathfrak{g}_{1 \star}\right]_{(a, b)}\right) \subset \mathbb{F}\left[\mathfrak{g}_{1 \star}\right]_{(a+1, b-1)} \oplus \mathbb{F}\left[\mathfrak{g}_{1 \star}\right]_{(a-1, b+1)} .
$$

Of course, if $a=0$ or $b=0$, then the summand with index $a-1$ or $b-1$ does not occur in the right-hand side. One can write $\mathcal{D}_{x}=\mathcal{D}_{x}^{+}+\mathcal{D}_{x}^{-}$, where

$$
\mathcal{D}_{x}^{+}\left(\mathbb{F}\left[\mathfrak{g}_{1 \star}\right]_{(a, b)}\right) \subset \mathbb{F}\left[\mathfrak{g}_{1 \star}\right]_{(a+1, b-1)} \text { and } \mathcal{D}_{x}^{-}\left(\mathbb{F}\left[\mathfrak{g}_{1 \star}\right]_{(a, b)}\right) \subset \mathbb{F}\left[\mathfrak{g}_{1 \star}\right]_{(a-1, b+1)} .
$$

Since $\mathcal{D}_{x}(f)=0$, we have $\mathcal{D}_{x}^{+}\left(f^{\bullet}\right)=0$ and $\mathcal{D}_{x}^{-}\left(f_{\bullet}\right)=0$. It also follows from $(4 \cdot 1)$ that $\mathcal{D}_{x}^{+}$ (resp. $\mathcal{D}_{x}^{-}$) is the derivation corresponding to $x$, as an element of $\mathfrak{g}_{01}^{a} \subset \mathfrak{k}$, in the algebra $\mathbb{F}\left[\mathfrak{g}_{01} \propto \mathfrak{g}_{11}\right]$ (resp. $\left.\mathbb{F}\left[\mathfrak{g}_{11} \propto \mathfrak{g}_{10}\right]\right)$.

Corollary 4.14. The algebras $\mathbb{F}[V]^{K}$ and $\mathbb{F}\left[V^{*}\right]^{K}$ are bi-graded.

Proof. The previous proof shows that if $f \in \mathbb{F}[V]^{K}$, then also $f^{\bullet} \in \mathbb{F}[V]^{K}$. Then one considers $f-f^{\bullet}$, etc.

Remark 4.15. Set gr $\bullet\left(\mathbb{F}\left[\mathfrak{g}_{1 \star}\right]^{G_{0 \star}}\right)=\left\{f^{\bullet} \mid f \in \mathbb{F}\left[\mathfrak{g}_{1 \star}\right]^{G_{0 \star}}\right\} \subset \mathbb{F}[V]^{K}$. We already know that $\operatorname{trdeg} \mathbb{F}(V)^{K}=\operatorname{trdeg} \mathbb{F}\left(\mathfrak{g}_{1 \star}\right)^{G_{0 \star}}=\operatorname{dim}\left(\mathfrak{g}_{1 \star} / / G_{0 \star}\right)$ and $\mathbb{F}(V)^{K}$ is the fraction field of $\mathbb{F}[V]^{K}$. Therefore, one might expect that $\operatorname{gr} \bullet\left(\mathbb{F}\left[\mathfrak{g}_{1 \star}\right]^{G_{0 \star}}\right)=\mathbb{F}[V]^{K}$ in good cases. Indeed, it often happens that there is a set of basic invariants $f_{1}, \ldots, f_{m} \in \mathbb{F}\left[\mathfrak{g}_{1 \star}\right]^{G_{0 \star}}$ such that $f_{1}^{\bullet}, \ldots, f_{m}^{\bullet}$ are algebraically independent. But, unlike the case of the coadjoint representation of $K$, 
this does not guarantee that $f_{1}^{\bullet}, \ldots, f_{m}^{\bullet}$ generate $\mathbb{F}[V]^{K}$ (cf. [17, Theorem 4.2]). True generators of $\mathbb{F}[V]^{K}$ can have smaller degrees. The reason is that, for the $K$-module $V$, the complement of the set of $K$-regular points may contain a divisor. (By [17, Theorem 3.3], this cannot happen for the coadjoint representation of a $\mathbb{Z}_{2}$-contraction.)

\section{INVARIANTS OF CERTAIN DEGENERATED ISOTROPY REPRESENTATIONS}

We continue to work with a quaternionic decomposition (2.2). In Section 4, we considered a good situation in which a CSS $\mathfrak{c}_{10} \subset \mathfrak{g}_{10}$ is also a CSS in $\mathfrak{g}_{1 \star}$. This is equivalent to that

$\mathfrak{z}_{\mathfrak{g}}\left(\mathfrak{c}_{10}\right) \cap \mathfrak{g}_{1 \star}=\mathfrak{c}_{10}$. Using Theorem 4.5, one then obtains a description of $\mathbb{F}\left[\mathfrak{g}_{10} \propto \mathfrak{g}_{11}\right]^{N_{01}}$, etc. In this section, we consider a less restrictive condition that $\mathfrak{c}_{10}$ contains $G_{0 \star}$-regular elements of $\mathfrak{g}_{1 \star}$. In other words,

$$
\mathfrak{z}_{\mathfrak{g}}\left(\mathfrak{c}_{10}\right) \cap \mathfrak{g}_{1 \star} \text { is a CSS in } \mathfrak{g}_{1 \star} \cdot
$$

Then $\mathfrak{d}:=\mathfrak{z}_{\mathfrak{g}}\left(\mathfrak{c}_{10}\right) \cap \mathfrak{g}_{1 \star}=\mathfrak{c}_{10} \oplus \mathfrak{a}$ for some subspace $\mathfrak{a} \subset \mathfrak{g}_{11}$. Recall that the generalised Weyl group for the CSS $\mathfrak{d} \subset \mathfrak{g}_{1 \star}$ is $\tilde{W}=N_{0 \star}(\mathfrak{d}) / Z_{0 \star}(\mathfrak{d})$, where $N_{0 \star}(\mathfrak{d})=\left\{s \in G_{0 \star} \mid s \cdot \mathfrak{d} \subset \mathfrak{d}\right\}$ and $Z_{0 \star}(\mathfrak{d})=\left\{s \in G_{0 \star} \mid s \cdot x=x \forall x \in \mathfrak{d}\right\}$. Set $m=\operatorname{dim} \mathfrak{d}$.

Obviously, the involution $\sigma_{2}$ preserves $\mathfrak{d}$ and the corresponding eigenspaces are $\mathfrak{c}_{10}$ and $\mathfrak{a}$.

Lemma 5.1. $\bar{\sigma}_{2}=\left.\sigma_{2}\right|_{\mathfrak{o}}$ normalises the group $\tilde{W} \subset G L(\mathfrak{d})$, i.e., $\bar{\sigma}_{2} \tilde{W} \bar{\sigma}_{2}^{-1}=\tilde{W}$.

Proof. If $g \in G_{0 \star}$, then $\sigma_{2}(g) \in G_{0 \star}$ as well, and $\sigma_{2}(g)$ acts on $\mathfrak{g}$ as composition $\sigma_{2} \circ g \circ \sigma_{2}^{-1}$. Therefore, if $g \in N_{0 \star}(\mathfrak{d})$, then also $\sigma_{2}(g) \in N_{0 \star}(\mathfrak{d})$.

Set $X=\overline{G_{0 \star} \cdot \mathfrak{c}_{10}} \subset \mathfrak{g}_{1 \star}$. Since $\overline{G_{00} \cdot \mathfrak{c}_{10}}=\mathfrak{g}_{10}$, we may as well define $X$ as $\overline{G_{0 \star} \cdot \mathfrak{g}_{10}}$.

Theorem 5.2. If condition (5.1) is satisfied, then $X$ is an irreducible complete intersection in $\mathfrak{g}_{1 \star}$ and the ideal of $X$ in $\mathbb{F}\left[\mathfrak{g}_{1 \star}\right], \mathcal{J}(X)$, is generated by some basic invariants in $\mathbb{F}\left[\mathfrak{g}_{1 \star}\right]^{G_{0 \star}}$.

Proof. Clearly, $X$ is irreducible. Since $\mathfrak{c}_{10}$ contains $G_{0 \star}$-regular elements of $\mathfrak{g}_{1 \star}$ and $\overline{G_{0 \star} \cdot \mathfrak{d}}=$ $\mathfrak{g}_{1 \star}$, we have $\operatorname{dim} \mathfrak{g}_{1 \star}-\operatorname{dim} X=\operatorname{dim} \mathfrak{d}-\operatorname{dim} \mathfrak{c}_{10}=\operatorname{dim} \mathfrak{a}$.

We know that $\bar{\sigma}_{2} \in G L(\mathfrak{d})$ is of finite order, normalises $\tilde{W}$, and has regular eigenvectors (in $\mathfrak{c}_{10}$ ). By [23, 6.3-6.5], this implies the following:

- The centraliser $W_{10}$ of $\bar{\sigma}_{2}$ in $\tilde{W}$ is reflection group in the $\bar{\sigma}_{2}$-eigenspace $\mathfrak{c}_{10}$.

- $\mathbb{F}\left[\mathfrak{c}_{10}\right]^{W_{10}}$ is generated by the restrictions to $\mathfrak{c}_{10}$ of some basic invariants in $\mathbb{F}[\mathfrak{d}]^{\tilde{W}}$. Namely, a set of basic invariants $\left\{f_{1}, \ldots, f_{m}\right\}$ can be chosen such that each $f_{i}$ is a $\bar{\sigma}_{2^{-}}$ eigenvector, say $\bar{\sigma}_{2}\left(f_{1}\right)=\varepsilon_{i} f_{i}$, where $\varepsilon_{i} \in\{1,-1\}$. Then $\mathbb{F}\left[\mathfrak{c}_{10}\right]^{W_{10}}$ is freely generated by $\left.f_{1}\right|_{\mathfrak{c}_{10}}$ such that $\varepsilon_{1}=1$. In particular, the restriction homomorphism $\mathbb{F}[\mathfrak{d}]^{\tilde{W}} \rightarrow \mathbb{F}\left[\mathfrak{c}_{10}\right]^{W_{10}}$ is onto.

- The eigenvalues of $\bar{\sigma}_{2}$ in $\mathfrak{d}$ are $\varepsilon_{1}, \ldots, \varepsilon_{m}$. 
It follows that there is a set of basic invariants $\left\{f_{1}, \ldots, f_{m}\right\}$ in $\mathbb{F}[\mathfrak{d}]^{\tilde{W}}$ such that $f_{1}, \ldots, f_{k}$ vanish on $\mathfrak{c}_{10}$, whereas the restrictions of $f_{k+1}, \ldots, f_{m}$ to $\mathfrak{c}_{10}$ freely generate $\mathbb{F}\left[\mathfrak{c}_{10}\right]^{W_{10}}$. Moreover, here $m-k=\operatorname{dim} \mathfrak{c}_{10}$ and $k=\operatorname{dim} \mathfrak{a}$.

Since $\mathbb{F}\left[\mathfrak{g}_{1 \star}\right]^{G_{0 \star}} \simeq \mathbb{F}[\mathfrak{d}]^{\tilde{W}}$, there are also $k$ basic invariants in $\mathbb{F}\left[\mathfrak{g}_{1 \star}\right]^{G_{0 \star}}$ that vanish on $X$. Let $F_{i}$ denote the $G_{0 *}$-invariant corresponding to $f_{i}$. Then $F_{1}, \ldots, F_{m}$ is a regular sequence in $\mathbb{F}\left[\mathfrak{g}_{1 \star}\right]$ and $\mathcal{V}\left(F_{1}, \ldots, F_{k}\right)$ is an unmixed variety of codimension dim a. Furthermore, $X \subset \mathcal{V}\left(F_{1}, \ldots, F_{k}\right)$ and $\operatorname{codim} X=k$. Hence $X$ is an irreducible component of $\mathcal{V}\left(F_{1}, \ldots, F_{k}\right)$. Our goal is to prove that the ideal $\left(F_{1}, \ldots, F_{k}\right)$ is prime. The first step is prove that $\mathcal{V}\left(F_{1}, \ldots, F_{k}\right)$ is irreducible.

Recall that the morphism $\pi: \mathfrak{g}_{1 \star} \rightarrow \mathfrak{g}_{1 \star} / / G_{0 \star}$ is equidimensional, and a generic fibre of $\pi$ is irreducible, since it is a (closed) $G_{0 \star}-$ orbit. If $Z$ is a $G_{0 \star}$-stable closed subset of $\mathfrak{g}_{1 \star}$, then $\pi(Z)$ is also closed [27,4.4]. The previous discussion on invariants of $\tilde{W}$ and $W_{10}$ shows that $\pi\left(\mathcal{V}\left(F_{1}, \ldots, F_{k}\right)\right)=\pi(X)=\pi\left(\mathfrak{c}_{10}\right)$ is an affine space of dimension $m-k$.

Let $Y$ be an irreducible component of $\mathcal{V}\left(F_{1}, \ldots, F_{k}\right)$. Since $\pi$ is equidimensional, $\operatorname{dim} X=\operatorname{dim} Y$, and $\pi(Y) \subset \pi\left(\mathcal{V}\left(F_{1}, \ldots, F_{k}\right)\right)=\pi(X)$, we must have $\pi(X)=\pi(Y)$. Moreover, for generic $\eta=\pi(x) \in \pi(Y)\left(x \in \mathfrak{c}_{10}\right)$, we have $\pi^{-1}(\eta)=G_{0 \star} \cdot x \subset X$. Thus, $Y=X$ and $X=\mathcal{V}\left(F_{1}, \ldots, F_{k}\right)$ is irreducible.

By [10, Lemma 4], the primeness of the ideal $\left(F_{1}, \ldots, F_{k}\right)$ will follow from the fact that there is a point $y \in X=\mathcal{V}\left(F_{1}, \ldots, F_{k}\right)$ such that the differentials $d F_{1}, \ldots, d F_{k}$ are linearly independent at $y$. Write $(d F)_{y}$ for the value of $d F$ at $y$. By assumption, $X$ contains a $G_{0 \star^{-}}$ regular semisimple element $x$, and it is known that even $\left(d F_{1}\right)_{x}, \ldots,\left(d F_{m}\right)_{x}$ are linearly independent [11].

Remark 5.3. Using the fact that $\left(d F_{1}\right)_{x}, \ldots,\left(d F_{k}\right)_{x}$ are linearly independent for any $G_{0 \star^{-}}$ regular point of $x \in X$, one easily proves that $X$ is non-singular in codimension 2 and therefore $X$ is normal. (A similar argument in the context of adjoint representations is found in $[19, \S 5]$.)

Keep the previous notation, i.e., $m=\operatorname{rk}\left(G / G_{0 \star}\right), X=\overline{G_{0 \star} \cdot \mathfrak{c}_{10}}$, and $F_{1}, \ldots, F_{m} \in \mathbb{F}\left[\mathfrak{g}_{1 \star}\right]^{G_{0 \star}}$ are the basic invariants such that $\mathcal{J}(X)=\left(F_{1}, \ldots, F_{k}\right)$ and $\mathbb{F}[X]^{G_{0 \star}}=\mathbb{F}\left[F_{k+1}, \ldots, F_{m}\right]$. Recall that $m-k=\operatorname{dim} \mathfrak{c}_{10}$ and $k=\operatorname{dim} \mathfrak{a}$. We wish to describe the invariants of the isotropy representation $\left(K: V=\mathfrak{g}_{10} \propto \mathfrak{g}_{11}\right)$, which is a contraction of $\left(G_{0 \star}: \mathfrak{g}_{1 \star}\right)$.

As in Section 4, we consider bi-homogeneous components of $F_{i}$ with respect to the sum $\mathfrak{g}_{1 \star}=\mathfrak{g}_{10} \oplus \mathfrak{g}_{11}$. Recall that $F_{i}^{\bullet}$ stands for the bi-homogeneous component of maximal degree with respect to $\mathfrak{g}_{10}$, and $F_{i}^{\bullet} \in \mathbb{F}\left[\mathfrak{g}_{10} \propto \mathfrak{g}_{11}\right]^{G 00 \times N_{01}}$ by Prop. 4.13.

Theorem 5.4. Suppose that condition (5.1) is satisfied.

(i) The field $\mathbb{F}\left(\mathfrak{g}_{10} \propto \mathfrak{g}_{11}\right)^{N_{01}}$ is generated by the coordinate functions on $\mathfrak{g}_{10}$ and the polynomials $F_{i}^{\bullet}, i=1, \ldots, k$. 
(ii) Suppose that $\left(d F_{1}\right)_{e}, \ldots,\left(d F_{k}\right)_{e}$ are linearly independent for a $G_{00}$-regular nilpotent element $e \in \mathfrak{g}_{10}$. Then

- the algebra $\mathbb{F}\left[\mathfrak{g}_{10} \propto \mathfrak{g}_{11}\right]^{N_{01}}$ is freely generated by the coordinate functions on $\mathfrak{g}_{10}$ and the polynomials $F_{i}^{\bullet}, i=1, \ldots, k$;

- the algebra $\mathbb{F}\left[\mathfrak{g}_{10} \propto \mathfrak{g}_{11}\right]^{G_{00} \ltimes N_{01}}$ is freely generated by the basic invariants in $\mathbb{F}\left[\mathfrak{g}_{10}\right]^{G_{00}}$ and the polynomials $F_{i}^{\bullet}, i=1, \ldots, k$.

Proof. We are only interested in basic invariants $F_{i}$ with $i \leqslant k$. Let $\operatorname{deg} F_{i}=n_{i}$. Since $F_{i}$ vanishes on $X$ and $\mathfrak{g}_{10}=\overline{G_{00} \cdot \mathfrak{c}_{10}} \subset X$, the bi-homogeneous component of degree $\left(n_{i}, 0\right)$ is trivial.

By (5.1), $\mathfrak{g}_{10}$ contains $G_{0 \star}$-regular semisimple elements. If $s \in \mathfrak{g}_{10}$ is such an element, then $\left(d F_{1}\right)_{s}, \ldots,\left(d F_{k}\right)_{s}$ are linearly independent. The fact that $\left(d F_{i}\right)_{s}$ is nonzero for some $s \in \mathfrak{g}_{10}$ implies that each $F_{i}$ has a bi-homogeneous component of degree $\left(n_{i}-1,1\right)$, which thereby is equal to $F_{i}^{\bullet}$. Since this bi-homogeneous component is linear with respect to $\mathfrak{g}_{11}$, it can be written as

$$
F_{i}^{\bullet}\left(y_{0}, y_{1}\right)=\kappa\left(\mathcal{F}_{i}\left(y_{0}\right), y_{1}\right)
$$

where $\mathcal{F}_{i}: \mathfrak{g}_{01} \rightarrow \mathfrak{g}_{11}$ is a polynomial mapping of degree $n_{i}-1$. Then

$$
\left(d F_{i}\right)_{y_{0}}=\mathcal{F}_{i}\left(y_{0}\right) .
$$

As $F_{i}^{\bullet}$ is $G_{00}$-invariant, the mapping $\mathcal{F}_{i}$ must be $G_{00}$-equivariant. It follows from Equations (5.2) and (4.1) that the $N_{01}$-invariance of $\mathcal{F}_{i}^{\bullet}$ is equivalent to that $\mathcal{F}_{i}\left(y_{0}\right)$ commutes with $y_{0}$. Recall that $\mathfrak{g}_{11}$ is a $K$-stable subspace of $V=\mathfrak{g}_{10} \propto \mathfrak{g}_{11}$ and the induced action of $N_{01}$ on $V / \mathfrak{g}_{11} \simeq \mathfrak{g}_{10}$ is trivial. Consider the polynomial mapping

$$
\psi: \mathfrak{g}_{10} \propto \mathfrak{g}_{11} \rightarrow\left(V / \mathfrak{g}_{11}\right) \times \mathbb{F}^{k} \simeq \mathfrak{g}_{10} \times \mathbb{F}^{k},
$$

defined by

$$
\psi\left(y_{0}, y_{1}\right)=\left(y_{0}, F_{1}^{\bullet}\left(y_{0}, y_{1}\right), \ldots, F_{k}^{\bullet}\left(y_{0}, y_{1}\right)\right)=\left(y_{0}, \kappa\left(\mathcal{F}_{1}\left(y_{0}\right), y_{1}\right), \ldots, \kappa\left(\mathcal{F}_{k}\left(y_{0}\right), y_{1}\right)\right) .
$$

Clearly the $N_{01}$-action on $\left(V / \mathfrak{g}_{11}\right) \times \mathbb{F}^{k}$ is trivial and $\psi$ is $N_{01}$-equivariant. Our ultimate goal is to prove that $\psi$ is the quotient by $N_{01}$. But we can perform it under the additional hypothesis in part (ii). Without extra hypotheses, we can only get the result on the field of $N_{01}$-invariants.

(i) Let $\Omega_{s} \subset \mathfrak{g}_{10}$ be the open subset of semisimple elements that are both $G_{0 \star}$-regular and $G_{00}$-regular. Condition (5.1) means that $\Omega_{s} \neq \varnothing$. Since the vectors $\mathcal{F}_{1}(\zeta), \ldots, \mathcal{F}_{k}(\zeta)$ are linearly independent for all $\zeta \in \Omega_{s}$, we have $\Omega_{s} \times \mathbb{F}^{k} \subset \operatorname{Im} \psi$. Hence $\psi$ is dominant. For $\left(y_{0}, z_{1}, \ldots, z_{k}\right) \in \mathfrak{g}_{10} \times \mathbb{F}^{k}$, the fibre

$$
\psi^{-1}\left(y_{0}, z_{1}, \ldots, z_{k}\right)=\left\{\left(y_{0}, y_{1}\right) \mid \kappa\left(\mathcal{F}_{j}\left(y_{0}\right), y_{1}\right)=z_{j}, 1 \leqslant j \leqslant k\right\}
$$


is an $N_{01}$-stable affine subspace of $\mathfrak{g}_{10} \propto \mathfrak{g}_{11}$. Furthermore, if $y_{0} \in \Omega_{s}$, then

$$
\operatorname{dim} \psi^{-1}\left(y_{0}, z_{1}, \ldots, z_{k}\right)=\operatorname{dim} \mathfrak{g}_{11}-k .
$$

On the other hand, if $\left(y_{0}, y_{1}\right) \in \psi^{-1}\left(y_{0}, z_{1}, \ldots, z_{k}\right)$, then

$$
N_{01} \cdot\left(y_{0}, y_{1}\right)=\left(y_{0}, y_{1}+\left[\mathfrak{g}_{01}, y_{0}\right]\right) \subset \psi^{-1}\left(y_{0}, z_{1}, \ldots, z_{k}\right)
$$

and $\operatorname{dim}\left[\mathfrak{g}_{01}, y_{0}\right]=\operatorname{dim}\left[\mathfrak{g}_{11}, y_{0}\right]=\operatorname{dim} \mathfrak{g}_{11}-\operatorname{dim} \mathfrak{z}_{\mathfrak{g}}\left(y_{0}\right)_{11}$. If $y_{0} \in \Omega_{s}$, then $\mathfrak{z}_{\mathfrak{g}}\left(y_{0}\right)_{10}$ is a CSS in $\mathfrak{g}_{10}$ and $\mathfrak{z}_{\mathfrak{g}}\left(y_{0}\right)_{1 \star}$ is a CSS in $\mathfrak{g}_{1 \star}$. Hence $\operatorname{dim} \mathfrak{z}_{\mathfrak{g}}\left(y_{0}\right)_{11}=\operatorname{dim} \mathfrak{a}=k$ and

$$
\operatorname{dim} N_{01} \cdot\left(y_{0}, y_{1}\right)=\operatorname{dim} \mathfrak{g}_{11}-k=\operatorname{dim} \psi^{-1}\left(y_{0}, z_{1}, \ldots, z_{k}\right) .
$$

As the orbits of a unipotent group on affine varieties are closed [21, Theorem 2] and isomorphic to affine spaces, we obtain $\psi^{-1}\left(y_{0}, z_{1}, \ldots, z_{k}\right)=N_{01} \cdot\left(y_{0}, y_{1}\right)$ for $y_{0} \in \Omega_{s}$, i.e., almost all fibres of $\psi$ are just $N_{01}$-orbits.

By [27, Lemma 2.1], this means that the coordinates on $\mathfrak{g}_{10}$ and $F_{1}^{\bullet}, \ldots, F_{k}^{\bullet}$ generate the field $\mathbb{F}\left(\mathfrak{g}_{10} \propto \mathfrak{g}_{11}\right)^{N_{01}}$.

(ii) We wish is to apply the Igusa lemma [16, Lemma 6.1] to $\psi$. As the target variety $\mathfrak{g}_{10} \times \mathbb{F}^{k}$ is normal and generic fibres of $\psi$ are $N_{01}$-orbits, it remains to check that $\operatorname{Im} \psi$ contains an open subset of $\mathfrak{g}_{10} \times \mathbb{F}^{k}$ whose complement is of codimension at least 2 .

Let $\widetilde{\Omega} \subset \mathfrak{g}_{10}$ be the open set of all $G_{00}$-regular elements (i.e., not only semisimple ones!). It follows from [11] that codim $\left(\mathfrak{g}_{10} \backslash \widetilde{\Omega}\right) \geqslant 2$. Hence it would be sufficient to have that $\widetilde{\Omega} \times \mathbb{F}^{k} \subset \operatorname{Im} \psi$. This, in turn, would follow from the fact that

$(\diamond) \quad$ the differentials $\left(d F_{1}\right)_{\eta}, \ldots,\left(d F_{k}\right)_{\eta}$ are linearly independent for all $\eta \in \widetilde{\Omega}$.

An obstacle is that if $\eta \in \widetilde{\Omega}$, then $\eta$ is not necessarily $G_{0 \star}$-regular. Therefore, we need the assumption that $\left(d F_{1}\right)_{e}, \ldots,\left(d F_{k}\right)_{e}$ are linearly independent for a nilpotent $e \in \widetilde{\Omega}$. Then a standard deformation argument guarantees us condition $(\diamond)$.

Thus, the assumptions of the Igusa lemma are satisfied and the assertion on the algebra of $N_{01}$-invariants follows. The assertion on $G_{00} \ltimes N_{01}$-invariants stems form the fact that the $\mathcal{F}_{i}^{\bullet '}$ are already $G_{00}$-invariant.

Remark 5.5. 1) It might be true that (5.1) already implies that $\left(d F_{1}\right)_{e}, \ldots,\left(d F_{k}\right)_{e}$ are linearly independent for a $G_{00}$-regular nilpotent $e \in \mathfrak{g}_{01}$. Such a phenomenon is observed in several examples. But we unable to either prove or disprove this as yet.

2) Although the algebra $\mathbb{F}\left[\mathfrak{g}_{10} \propto \mathfrak{g}_{11}\right]^{N_{01}}$ is polynomial in Theorem 5.4(ii), the quotient morphism

$$
\pi_{N_{01}}: \mathfrak{g}_{10} \propto \mathfrak{g}_{11} \rightarrow\left(\mathfrak{g}_{10} \propto \mathfrak{g}_{11}\right) / / N_{01} \simeq \mathfrak{g}_{10} \times \mathbb{F}^{k}
$$

is not equidimensional (unless $k=0$, i.e., we are in the situation of Theorem 4.5). Yet it might be true that the quotient morphism by $K=G_{00} \ltimes N_{01}$

$$
\pi_{K}: \mathfrak{g}_{10} \propto \mathfrak{g}_{11} \rightarrow\left(\mathfrak{g}_{10} \propto \mathfrak{g}_{11}\right) / /\left(G_{00} \ltimes N_{01}\right) \simeq\left(\mathfrak{g}_{10} / / G_{00}\right) \times \mathbb{F}^{k} \simeq \mathbb{F}^{m}
$$


is equidimensional. However, our methods for verifying equidimensionality require an information on the dimension of the linear span of $\left\{\left(d F_{1}\right)_{v}, \ldots,\left(d F_{k}\right)_{v}\right\}$ for nilpotent elements of $v \in \mathfrak{g}_{10}$, which is difficult to infer in general (cf. proof of [17, Theorem 5.3]).

There is a sufficient condition that guarantees that Theorem 5.4 applies in full strength.

Proposition 5.6. Let $e \in \mathfrak{g}_{10}$ be a $G_{00}$-regular nilpotent element. If $e$ is also $G_{0 \star}$-regular as an element of $\mathfrak{g}_{1 *}$, then then all the assumptions of Theorem 5.4 are satisfied, and hence both $\mathbb{F}\left[\mathfrak{g}_{10} \propto \mathfrak{g}_{11}\right]^{N_{01}}$ and $\mathbb{F}\left[\mathfrak{g}_{10} \propto \mathfrak{g}_{11}\right]^{G_{00} \propto N_{01}}$ are polynomial algebras.

Proof. Let $\{e, h, f\} \subset \mathfrak{g}_{\star 0}$ be a normal $\mathfrak{s l}_{2}$-triple. If $e$ is both $G_{00}$-regular and $G_{0 \star}$-regular, then the same is true for the semisimple element $e+f \in \mathfrak{g}_{10}$ [11]. Hence condition (5.1) is satisfied. It was also explained above that the $G_{0 \star}$-regularity of $e$ implies the linear independence of the differentials for all basic invariants in $\mathbb{F}\left[\mathfrak{g}_{1 \star}\right]^{G{ }_{0 \star}}$.

We consider below some applications of Theorem 5.4(ii).

Example 5.7. Let $\left\{\vartheta, \vartheta^{\prime}, \mu\right\}$ be a canonical triple of involutions of $\mathfrak{g}$, i.e., $\vartheta, \vartheta^{\prime}$ are of maximal rank and $\mu$ is quasi-maximal. The corresponding quaternionic decomposition

$$
\mathfrak{g}=\begin{array}{c:c}
\mathfrak{g}_{00} & \mathfrak{g}_{01} \\
\hdashline \mathfrak{g}_{10} \ldots . . & \ldots \ldots \ldots \\
\hdashline \vartheta^{\prime} & \mathfrak{g}_{11}
\end{array}
$$

has a number of good properties, see Proposition 2.2 and Remark 2.3. We wish to describe the algebras of invariants for all degenerated isotropy representations. By Corollary 3.5, there are at least four coincidence of CSS; hence the invariants for the respective degenerated isotropy representations are given by Theorem 4.9. If $\vartheta$ is inner, i.e., $\left\{\vartheta, \vartheta^{\prime}, \mu\right\}$ is actually a triad, then all little and big CSS are Cartan subalgebras and the invariants of all six degenerations are described by Theorem 4.9(iii). But, if $\vartheta$ is not inner, then there are two degenerated isotropy representations, $\left(G_{00} \ltimes N_{01}: \mathfrak{g}_{10} \propto \mathfrak{g}_{11}\right)$ and $\left(G_{00} \ltimes N_{10}: \mathfrak{g}_{01} \propto \mathfrak{g}_{11}\right)$, where Theorem 4.9 does not apply. We show that the hypotheses of Theorem 5.4 are satisfied for them, and hence the their algebras of invariants are also polynomial.

Assume below that $\vartheta$ is not inner, so that $\vartheta$ and $\mu$ are not conjugate. Then $k_{0} \neq 0$ and

$$
\operatorname{dim} \mathfrak{g}_{00}=\frac{\operatorname{dim} U-k_{1}}{2}, \operatorname{dim} \mathfrak{g}_{01}=\operatorname{dim} \mathfrak{g}_{10}=\operatorname{dim} \mathfrak{g}_{00}+k_{1}, \operatorname{dim} \mathfrak{g}_{11}=\operatorname{dim} \mathfrak{g}_{00}+\operatorname{rk} \mathfrak{g}
$$

(see the proof of Prop. 2.2). We also have $\mathrm{rk} \mathfrak{g}^{\vartheta}=\operatorname{rk} \mathfrak{g}^{\vartheta^{\prime}}=k_{1}$ (Proposition 1.3). As $\vartheta$ is of maximal rank, any CSS $\mathfrak{c}_{1 \star} \subset \mathfrak{g}_{1 \star}$ is a Cartan subalgebra of $\mathfrak{g}$. Recall that $\mathbb{F}\left[\mathfrak{g}_{1 \star}\right]^{G_{0 \star}} \simeq$ $\mathbb{F}\left[\mathfrak{c}_{1 \star}\right] \tilde{W}$, where $\tilde{W}$ is the generalised Weyl group. In this case, $\tilde{W}$ coincides with the usual Weyl group of $\mathfrak{g}$ with respect to $\mathfrak{c}_{1 \star}$. Hence $\mathbb{F}\left[\mathfrak{g}_{1 \star}\right]^{G_{0 \star}} \simeq \mathbb{F}[\mathfrak{g}]^{G}$. Let $\mathfrak{c}_{10}$ be a CSS in $\mathfrak{g}_{10}$. Then $\operatorname{dim} \mathfrak{c}_{10}=k_{1}=\operatorname{rk} \mathfrak{g}-k_{0}$. Hence $\mathfrak{c}_{10}$ cannot be a CSS in $\mathfrak{g}_{1 \star}$. However, $\mathfrak{c}_{10}$ does contain regular semisimple elements of $\mathfrak{g}$ (see the proof of Prop. 2.2), i.e., (5.1) is satisfied. 
In this situation, the number $k$ occurring in Theorems 5.2 and 5.4 is $k_{0}$ and basic invariants $F_{1}, \ldots, F_{k_{0}}$ are precisely the basic invariants in $\mathbb{F}[\mathfrak{g}]^{G}$ of odd degrees. (Recall that we have defined $k_{0}$ as the number of even exponents of $\mathfrak{g}$.) If $e \in \mathfrak{g}_{10}$ is $G_{00}$-regular nilpotent, then $e$ is also regular nilpotent in $\mathfrak{g}_{\star 0}=\mathfrak{g}^{\vartheta^{\prime}}$, since this involution of $\mathfrak{g}_{\star 0}$ is of maximal rank, see [1]. Therefore, the hypothesis of Theorem 5.4(ii) can be restated as follows:

Let $\vartheta$ be an (outer) involution of maximal rank and e a regular nilpotent element of $\mathfrak{g}^{\vartheta}$. Let $F_{1}, \ldots, F_{k_{0}} \in \mathbb{F}[\mathfrak{g}]^{G}$ be the basic invariants of odd degrees. Then $\left(d F_{1}\right)_{e}, \ldots,\left(d F_{k_{0}}\right)_{e}$ are linearly independent.

We verify this for all relevant simple Lie algebras.

$\left.\mathrm{a}_{1}\right) \mathfrak{g}=\mathfrak{s l}_{2 n+1}$ and $\mathfrak{g}^{\vartheta}=\mathfrak{s o}_{2 n+1}$. Here $e$ is regular in the whole of $\mathfrak{g}$.

$\left.\mathrm{a}_{2}\right) \mathfrak{g}=\mathfrak{s l}_{2 n}$ and $\mathfrak{g}^{\vartheta}=\mathfrak{s o}_{2 n}$. The partition of $e$ is $(2 n-1,1)$, i.e., $e$ is a subregular nilpotent element of $\mathfrak{g}$. Let $f_{i}$ be a basic invariant of degree $i(i=2,3, \ldots, 2 n)$. It is known that, for a subregular nilpotent element $e_{,}\left(d f_{2 n}\right)_{e}=0$ (i.e., for the basic invariant of maximal degree), while the remaining differentials are linearly independent [20, Lemma 5.1]. This remaining set contains all basic invariants of odd degree.

$\left.\mathrm{a}_{3}\right) \mathfrak{g}=\mathfrak{s o}_{4 n+2}$ and $\mathfrak{g}^{\vartheta}=\mathfrak{s o}_{2 n+1} \times \mathfrak{s o}_{2 n+1}$. The partition of $e$ is $(2 n+1,2 n+1)$ and $k_{0}=1$. The only basic invariant of odd degree is the pfaffian $\mathrm{P}$. One verifies that, for a nilpotent element $v,(d \mathrm{P})_{v}=0$ if and only if the partition of $v$ has at least three nonzero parts [20, Lemma 4.1.1].

$\left.\mathrm{a}_{4}\right) \mathfrak{g}=\mathbf{E}_{6}$ and $\mathfrak{g}^{\vartheta}=\mathfrak{s p}_{8}$. Here $k_{0}=2, \operatorname{deg} F_{1}=5$ and $\operatorname{deg} F_{2}=9$. As $e$ appears to be subregular in $\mathfrak{g}$, we can again use [20, Lemma 5.1].

Thus, in case of canonical decompositions, we can describe the algebras of invariants for all six degenerated isotropy representations.

Example 5.8. Let $\mathfrak{g}$ be of type $\mathbf{E}_{6}$. According to [9, Table 1], there are three commuting involutions with the following fixed-point subalgebras:

$$
\sigma_{1}, \sigma_{2}: \mathbf{F}_{4} ; \quad \sigma_{3}: \mathbf{D}_{5} \oplus \mathfrak{t}_{1} .
$$

As $\left\{\sigma_{1}, \sigma_{2}\right\}$ is supposed to be a dyad, this can also be verified using the restricted root system for $\mathbf{E}_{6} / \mathbf{F}_{4}$, which is reduced and of type $\mathbf{A}_{2}$ (see Section 2). Here $\mathfrak{g}_{00}=\mathfrak{s o}_{9}$ and the $\mathfrak{g}_{00}$-modules $\mathfrak{g}_{i j}$ are:

$$
\mathfrak{g}_{01} \simeq \mathfrak{g}_{10} \simeq \mathrm{R}_{\varpi_{4}}, \mathfrak{g}_{11} \simeq \mathrm{R}_{\varpi_{1}}+\mathrm{R}_{0},
$$

where $\varpi_{i}$ 's are fundamental weights of $\mathfrak{s o}_{9}$ and $R_{\lambda}$ stands for the simple module with highest weight $\lambda$. Hence $\operatorname{dim} \mathfrak{g}_{01}=\operatorname{dim} \mathfrak{g}_{10}=16$ and $\operatorname{dim} \mathfrak{g}_{11}=10$. Here $\operatorname{dim} \mathfrak{c}_{10}=\operatorname{dim} \mathfrak{c}_{01}=$ 1 and $\operatorname{dim} \mathfrak{c}_{11}=2$. By Theorem 3.7, $\mathfrak{c}_{11}$ is also a CSS in $\mathfrak{g}_{1 \star}$ or $\mathfrak{g}_{\star 1}$; and these are the only coincidences of CSS here. In view of these coincidence and Theorem 4.9(ii), the algebra of invariants for two isomorphic degenerated isotropy representations, $\left(G_{00} \ltimes N_{01}: \mathfrak{g}_{11} \propto \mathfrak{g}_{10}\right)$ and $\left(G_{00} \ltimes N_{10}: \mathfrak{g}_{11} \propto \mathfrak{g}_{01}\right)$, is readily described:

$$
\mathbb{F}\left[\left(\mathrm{R}_{\varpi_{1}}+\mathrm{R}_{0}\right) \propto \mathrm{R}_{\varpi_{4}}\right]^{\mathrm{Spin}_{9} \times \exp \left(\mathrm{R}_{\varpi_{4}}^{a}\right)} \simeq \mathbb{F}\left[\mathrm{R}_{\varpi_{1}}+\mathrm{R}_{0}\right]^{\mathrm{Spin}_{9}} .
$$


Therefore, this algebra has the basic invariants of degree 1 and 2 . The representation

$$
\left(\operatorname{Spin}_{9} \ltimes \exp \left(R_{\varpi_{4}}^{a}\right):\left(R_{\varpi_{1}}+R_{0}\right) \propto R_{\varpi_{4}}\right)
$$

is a degeneration of the isotropy representation of the symmetric space $\mathbf{E}_{6} / \mathbf{F}_{4}$, i.e., of $\left(F_{4}: R\right)$, where $\operatorname{dim} R=26$ and the degrees of basic invariants are 2 and 3 . The other degeneration of the same isotropy representation is

$$
\left(\operatorname{Spin}_{9} \ltimes \exp \left(\mathrm{R}_{\varpi_{4}}^{a}\right): \mathrm{R}_{\varpi_{4}} \propto\left(\mathrm{R}_{\varpi_{1}}+\mathrm{R}_{0}\right)\right) .
$$

Here we can exploit the fact that any $G_{00}$-regular nilpotent element in $\mathfrak{g}_{10} \simeq \mathrm{R}_{\varpi_{4}}$ appears to be $G_{0 \star}$-regular in $\mathfrak{g}_{1 \star} \simeq \mathrm{R}$. Indeed, using the Satake diagram for the symmetric pair $\left(\mathbf{F}_{4}, \mathfrak{s o}_{9}\right)$, one verifies that the $\operatorname{Spin}_{9}$-regular nilpotent element of $\mathfrak{g}_{10}$ belong to the nilpotent $\mathbf{F}_{4}$-orbit $\mathcal{O}$, denoted $\tilde{\mathbf{A}}_{2}$. On the other hand, using the Satake diagram for the symmetric pair $\left(\mathbf{E}_{6}, \mathbf{F}_{4}\right)$, one verifies that the $\mathbf{F}_{4}$-regular nilpotent element of $\mathfrak{g}_{1 \star}$ belongs to the nilpotent $\mathbf{E}_{6}$-orbit $\tilde{\mathcal{O}}$, denoted $2 \mathbf{A}_{2}$. It is not hard to verify using the respective weighted Dynkin diagrams that $\tilde{\mathcal{O}} \cap \mathfrak{f}_{4}=\mathcal{O}$.

Since $\operatorname{dim} \mathfrak{c}_{1 \star}-\operatorname{dim} \mathfrak{c}_{10}=1, X=\overline{G_{0 \star} \cdot \mathfrak{g}_{10}}$ is a hypersurface in $\mathfrak{g}_{1 \star}$, and this hypersurface must be the zero set of the basic invariant of degree 3 in $\mathbb{F}[R]^{\mathrm{F}_{4}}$. Finally, applying Theorem 5.4 and Prop. 5.6 shows that $\mathbb{F}\left[R_{\varpi_{4}} \propto\left(R_{\varpi_{1}}+R_{0}\right)\right]^{\operatorname{Sin}_{9} \ltimes \exp \left(R_{\varpi_{4}}^{a}\right)}$ is a polynomial algebra with basic invariants of degree 2 and 3 .

Thus, the dual representations (5.3) and (5.4) have polynomial algebras of invariants, but the degrees of basic invariants are different.

Set $V=\left(\mathrm{R}_{\varpi_{1}}+\mathrm{R}_{0}\right) \propto \mathrm{R}_{\varpi_{4}}, V^{*}=\mathrm{R}_{\varpi_{4}} \propto\left(\mathrm{R}_{\varpi_{1}}+\mathrm{R}_{0}\right)$, and $K=\operatorname{Spin}_{9} \ltimes \exp \left(\mathrm{R}_{\varpi_{4}}^{a}\right)$. Since $\operatorname{dim} V / / K=\operatorname{dim} V^{*} / / K=2$ and $K$ has no rational characters, one easily derives that both morphisms $\pi_{V}: V \rightarrow V / / K \simeq \mathbb{A}^{2}$ and $\pi_{V^{*}}: V^{*} \rightarrow V^{*} / / K \simeq \mathbb{A}^{2}$ are equidimensional; hence $\mathbb{F}[V]$ is a free $\mathbb{F}[V]^{K}$-module, and likewise for $V^{*}$.

\section{PROBlems AND OBSERVATIONS}

6.1. In [17], I suggested that the coadjoint representation of a $\mathbb{Z}_{2}$-contraction of $\mathfrak{g}$ always has a polynomial algebra of invariants. However, recent work of O. Yakimova [28] demonstrates that this is not always the case. Therefore, there ought to be quaternionic decompositions of a simple Lie algebra $\mathfrak{g}$ such that some degenerated isotropy representations $\left(G_{00} \ltimes N_{\beta}: \mathfrak{g}_{\alpha} \propto \mathfrak{g}_{\gamma}\right)$ do not have a polynomial algebra of invariants. On the other

hand, results of Sections 4 and 5 show that in many cases the algebra $\mathbb{F}\left[\mathfrak{g}_{\alpha} \propto \mathfrak{g}_{\gamma}\right]^{G_{00} \ltimes N_{\beta}}$ is polynomial. This raises the following

Question 1. What are more precise necessary and/or sufficient conditions for $\mathbb{F}\left[\mathfrak{g}_{\alpha} \propto \mathfrak{g}_{\gamma}\right]^{G_{00} \propto N_{\beta}}$ to be polynomial? 
In Section 4, we proved that if a CSS $\mathfrak{c}_{\alpha} \subset \mathfrak{g}_{\alpha}$ is also a CSS in $\mathfrak{g}_{\alpha} \oplus \mathfrak{g}_{\gamma}$, then $\mathbb{F}\left[\mathfrak{g}_{\alpha} \propto \mathfrak{g}_{\gamma}\right]$ is a

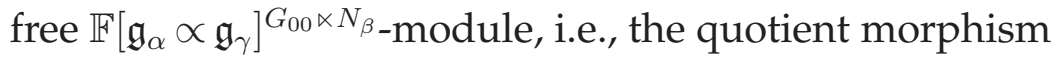

$$
\pi: \mathfrak{g}_{\alpha} \propto \mathfrak{g}_{\gamma} \rightarrow\left(\mathfrak{g}_{\alpha} \propto \mathfrak{g}_{\gamma}\right) / / G_{00} \ltimes N_{\beta} \simeq \mathbb{A}^{m}
$$

is equidimensional. This raises our next

Question 2. What are more precise necessary and/or sufficient conditions for $\mathbb{F}\left[\mathfrak{g}_{\alpha} \propto \mathfrak{g}_{\gamma}\right]$ to be a free $\mathbb{F}\left[\mathfrak{g}_{\alpha} \propto \mathfrak{g}_{\gamma}\right]^{G_{00} \ltimes N_{\beta}}$-module?

Of course, both Questions are prompted by the fact that the respective properties are always satisfied for the initial (big) isotropy representations, see Section 1.

6.2. Let $Q$ be an algebraic group with $\operatorname{Lie}(Q)=\mathfrak{q}$. Suppose that $\sigma$ is an involution of $Q$ and $Q_{0}$ is the identity component of $Q^{\sigma}$. Let $H$ be any subgroup between $Q_{0}$ and $Q^{\sigma}$ such that $Q / H$ is connected (this is always the case if $Q$ is connected). Then the algebra, $\mathcal{D}(Q / H)$, of left invariant differential operators on the homogeneous space $Q / H$ is commutative [22],[4]. Let $\mathfrak{q}=\mathfrak{q}_{0} \oplus \mathfrak{q}_{1}$ be the corresponding $\mathbb{Z}_{2}$-grading. The following is proved in [5, Lemma 4.2]:

$$
\text { If the algebra } \mathcal{S}\left(\mathfrak{q}_{1}\right)^{H}=\mathbb{F}\left[\mathfrak{q}_{1}^{*}\right]^{H} \text { is polynomial, then so is } \mathcal{D}(Q / H) \text {. }
$$

In fact, there is a canonical linear bijection between the two algebras that transforms the free generators of the former to the free generators of the latter.

Therefore, our results on quaternionic decompositions provide a description of invariant differential operators for certain degenerations of symmetric spaces of $G$. Recall that the group $K_{01}=G_{00} \ltimes N_{01}$ can be regarded as the identity component of a symmetric subgroup in two different ways:

a) $K_{01}$ is the identity component of $G\left\langle\sigma_{2}\right\rangle^{\sigma_{1}}$, and the corresponding isotropy representation is $\left(K_{01}: \mathfrak{g}_{10} \propto \mathfrak{g}_{11}\right)$;

b) $K_{01}$ is the identity component of $G\left\langle\left.\sigma_{3}\right|^{\sigma_{1}}\right.$, and the corresponding isotropy representation is $\left(K_{01}: \mathfrak{g}_{11} \propto \mathfrak{g}_{10}\right)$.

Taking into account that $\left(\mathfrak{g}_{10} \propto \mathfrak{g}_{11}\right)^{*}=\mathfrak{g}_{11} \propto \mathfrak{g}_{10}$, we obtain the following:

Claim 6.1. If $\mathbb{F}\left[\mathfrak{g}_{10} \propto \mathfrak{g}_{11}\right]^{K_{01}}$ is a polynomial algebra, then so is $\mathcal{D}\left(G\left\langle\sigma_{3}\right\rangle / K_{01}\right)$; if $\mathbb{F}\left[\mathfrak{g}_{11} \propto \mathfrak{g}_{10}\right]^{K_{01}}$ is a polynomial algebra, then so is $\mathcal{D}\left(G\left\langle\sigma_{2}\right\rangle / K_{01}\right)$.

It might be interesting to explore a relationship between the joint eigenfunctions for $\mathcal{D}\left(G / G_{0 \star}\right)$ and $\mathcal{D}\left(G\left\langle\sigma_{3}\right\rangle / K_{01}\right)$. Also, if $\mathfrak{c}_{10}$ is a CSS in $\mathfrak{g}_{01} \oplus \mathfrak{g}_{11}$, then $\mathbb{F}\left[\mathfrak{g}_{10} \propto \mathfrak{g}_{11}\right]^{K_{01}} \simeq$ $\mathbb{F}\left[\mathfrak{g}_{10}\right]^{G 00}$, which seems to suggest that there might be a direct relation between the polynomial algebras of differential operators $\mathcal{D}\left(G\left\langle\sigma_{3}\right\rangle / K_{01}\right)$ and $\mathcal{D}\left(G_{\star 0} / G_{00}\right)$. 
6.3. In [5], Gonzalez and Helgason determined the algebra of invariant differential operators for an interesting non-reductive symmetric pair $\left(Q, Q^{\sigma}\right)$. In their exposition, the (non-connected) group $Q$ is the semi-direct product of the orthogonal group $\mathcal{O}_{n}=\mathcal{O}(V)$ and the standard $\mathcal{O}(V)$-module $V$, i.e., using our notation, $\mathfrak{q}=\mathfrak{s o}(V) \ltimes V^{a}$ and $Q=\mathcal{O}(V) \ltimes$ $\exp \left(V^{a}\right)$. Then they consider an involution $\sigma$ of $Q$ such that $Q^{\sigma}=\left(\mathcal{O}\left(V_{1}\right) \ltimes \exp \left(V_{1}^{a}\right)\right) \times \mathcal{O}\left(V_{2}\right)$, where $V=V_{1} \oplus V_{2}$ is an orthogonal direct sum. Set $p=\operatorname{dim} V_{1}$, so that $n-p=\operatorname{dim} V_{2}$.

Our observation is that $Q / Q^{\sigma}$ is a degeneration of the symmetric space $\mathcal{O}_{n+1} / \mathcal{O}_{p+1} \times$ $\mathcal{O}_{n-p}$, and this degeneration is related to a quaternionic decomposition of $\mathfrak{g}=\mathfrak{s o}_{n+1}$. To get this, we assume that $\mathfrak{s o}_{n+1}=\mathfrak{s o}(\tilde{V})$ consists of usual skew-symmetric matrices of order $n+1$ with respect to a certain basis of $(n+1)$-dimensional space $\tilde{V}$ and consider the involutions $\sigma_{i}(i=1,2,3)$ defined by the diagonal matrices $s_{1}, s_{2}, s_{3} \in \mathcal{O}(\tilde{V})$ :

$$
s_{1}=\operatorname{diag}(\underbrace{1, \ldots, 1}_{p+1}, \underbrace{-1, \ldots,-1}_{n-p}), \quad s_{2}=\operatorname{diag}(\underbrace{1, \ldots, 1}_{p}, \underbrace{-1, \ldots,-1}_{n-p+1}), \quad s_{3}=s_{1} s_{2} .
$$

Let $V_{1}$ (resp. $V_{2}$ ) be the linear span of the first $p$ (resp. last $n-p$ ) basis vectors. Then $\mathfrak{g}_{00}=\mathfrak{s o}\left(V_{1}\right) \times \mathfrak{s o}\left(V_{2}\right)$ and the whole quaternionic decomposition is:

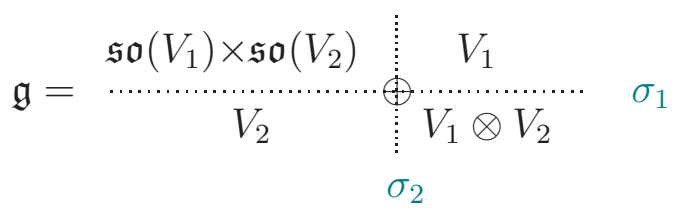

(i.e., $\mathfrak{g}_{10} \simeq V_{2}$ as $\mathfrak{g}_{00}$-module, etc.) Here $\tilde{V}=V_{1} \oplus V_{2} \oplus \mathbb{F}=V \oplus \mathbb{F}$, $\mathfrak{g}^{\sigma_{1}}=\mathfrak{s o}\left(V_{1} \oplus\right.$ $\mathbb{F}) \times \mathfrak{s o}\left(V_{2}\right), \mathfrak{g}^{\sigma_{2}}=\mathfrak{s o}\left(V_{2} \oplus \mathbb{F}\right) \times \mathfrak{s o}\left(V_{1}\right)$, and $\mathfrak{g}^{\sigma_{3}}=\mathfrak{s o}(V)$. In our notation, the Lie algebra $\operatorname{Lie}(Q)=\mathfrak{q}$ considered in [5] is nothing but $\mathfrak{g}\left\langle\sigma_{3}\right\rangle$; and Theorem 3.1 in [5] essentially means that, for $H:=\left(\mathcal{O}\left(V_{1}\right) \times \mathcal{O}\left(V_{2}\right)\right) \ltimes \exp \left(V_{1}^{a}\right)$, the algebra $\mathbb{F}\left[V_{2} \propto\left(V_{1} \otimes V_{2}\right)\right]^{H}$ is polynomial, of Krull dimension $\min \{p+1, n-p\}$. In our setting, the dimension formula is explained by Corollary 4.12 and the fact that $\operatorname{rk}\left(\mathcal{S O}_{n+1} / \mathcal{S O}_{p+1} \times \mathcal{S O}_{n-p}\right)=\min \{p+1, n-p\}$. The identity component of $H$ is

$$
K_{01}=\left(\mathcal{S O}\left(V_{1}\right) \times \mathcal{S O}\left(V_{2}\right)\right) \ltimes \exp \left(V_{1}^{a}\right),
$$

and one can prove that, for this degenerated isotropy representation, we have a materialisation of the phenomenon of Remark 4.15, i.e., gr $\bullet\left(\mathbb{F}\left[\mathfrak{g}_{1 \star}\right]^{G_{0 \star}}\right)=\mathbb{F}\left[V_{2} \propto\left(V_{1} \otimes V_{2}\right)\right]^{K_{01}}$. Note that $G_{0 \star}=\mathcal{S O}\left(V_{1} \oplus \mathbb{F}\right) \times \mathcal{S O}\left(V_{2}\right)$ and the basic invariants of $\mathbb{F}\left[\mathfrak{g}_{1 \star}\right]^{G_{0 \star}}$ have degrees

$$
\begin{cases}2,4, \ldots, 2 \min \{p+1, n-p\} & \text { if } p+1 \neq n-p, \\ 2,4, \ldots, 2 p, p+1 & \text { if } p+1=n-p .\end{cases}
$$

Therefore, the same is true for $\mathbb{F}\left[V_{2} \propto\left(V_{1} \otimes V_{2}\right)\right]^{K_{01}}$. Since $\operatorname{dim} \mathfrak{c}_{10}=1$ and $\operatorname{dim} \mathfrak{c}_{1 \star}=\min \{p+$ $1, n-p\}$, the coincidence of CSS occurs only if $n-p=1$. 
The advantage of using quaternionic decompositions is that we immediately get other interesting possibilities. For the dual $K_{01}$-module $\left(V_{1} \otimes V_{2}\right) \propto V_{2}$, we may compare the Cartan subspaces $\mathfrak{c}_{11} \subset V_{1} \otimes V_{2}$ and $\mathfrak{c}_{1 \star}$.

- Since $\operatorname{dim} \mathfrak{c}_{11}=\min \{p, n-p\}$ and $\operatorname{dim} \mathfrak{c}_{1 \star}=\min \{p+1, n-p\}$, we have a coincidence of CSS if and only if $n-p \leqslant p$, and then $\mathbb{F}\left[\left(V_{1} \otimes V_{2}\right) \propto V_{2}\right]^{K_{01}} \simeq \mathbb{F}\left[V_{1} \otimes V_{2}\right]^{\mathcal{S O}\left(V_{1}\right) \times \mathcal{S O}\left(V_{2}\right)}$. The latter is a polynomial algebra whose degrees of basic invariants are

$2,4, \ldots, 2(n-p)$, if $n-p<p ; \quad 2,4, \ldots, 2 p-2, p$, if $n-p=p$.

- For $p+1=n-p$, one can notice that a $G_{00}$-regular nilpotent element in $\mathfrak{g}_{11} \simeq V_{1} \otimes V_{2}$ is actually regular in the whole of $\mathfrak{g}$. Hence, Proposition 5.6 applies to $K_{01}:\left(V_{1} \otimes V_{2}\right) \propto V_{2}$ and hence $\mathbb{F}\left[\left(V_{1} \otimes V_{2}\right) \propto V_{2}\right]^{K_{01}}$ is polynomial.

- Even for $p+1<n-p$, we can prove using the 'contraction procedure' for $G_{0 \star^{-}}$ invariants that $\mathbb{F}\left[\left(V_{1} \otimes V_{2}\right) \propto V_{2}\right]^{K_{01}}$ is a polynomial algebra. The details will appear elsewhere.

As explained in Subsection 6.2, this implies that $\mathcal{D}\left(G\left\langle\sigma_{2}\right\rangle / K_{01}\right)$ is a polynomial algebra, where $G=\mathcal{S O}\left(V_{1} \oplus V_{2} \oplus \mathbb{F}\right)$ and $G\left\langle\sigma_{2}\right\rangle=\left(\mathcal{S O}\left(V_{1}\right) \times \mathcal{S O}\left(V_{2} \oplus \mathbb{F}\right)\right) \ltimes\left(V_{1} \otimes\left(V_{2} \oplus \mathbb{F}\right)\right)$.

Acknowledgements. Part of this work was done while I was able to use rich facilities of the Max-Planck Institut für Mathematik (Bonn).

\section{REFERENCES}

[1] Л.В. Антонян. О классификации однородных элементов $\mathbb{Z}_{2}$-градуированных полупростых алгебр Ли, Вестник Моск. Ун-та, Сер. Матем. Мех., № 2 (1982), 29-34 (Russian). English translation: L.V. ANTONYAN. On classification of homogeneous elements of $\mathbb{Z}_{2}$-graded semisimple Lie algebras, Moscow Univ. Math. Bulletin, 37 (1982), № 2, 36-43.

[2] A. Bialynicki-Birula. On homogeneous affine spaces of linear algebraic groups, Amer. J. Math. 85 (1963), 577-582.

[3] M. BulOIS. Sheets of symmetric Lie algebras and Slodowy slices, J. Lie Theory, 21 (2011), 1-54.

[4] M. Duflo. Opérateurs différentiels invariants sur un espace symétrique. C. R. Acad. Sci., Sér. A-B 289 (1979), no. 2, A135-A137.

[5] F. GONZALEZ and S. Helgason. Invariant differential operators on Grassmann manifolds, Adv. in Math., 60 (1986), 81-91.

[6] S. Helgason. "Differential geometry, Lie groups, and symmetric spaces”, New York-London: Academic Press, 1978.

[7] A. HELminCK and G. SCHWARZ. Orbits and invariants associated with a pair of commuting involutions, Duke Math. J., 106 (2001), 237-279.

[8] A. HELMinCK and G. SCHWARZ. Smoothness of quotients associated with a pair of commuting involutions, Canad. J. Math., 56 (2004), 945-962.

[9] A. Kollross. Exceptional $\mathbb{Z}_{2} \times \mathbb{Z}_{2}$-symmetric spaces, Pacific J. Math., 242, no. 1 (2009), 113-130.

[10] B. Kostant. Lie group representations in polynomial rings, Amer. J. Math., 85 (1963), 327-404.

[11] B. Kostant and S. RALLIS. Orbits and representations associated with symmetric spaces, Amer. J. Math., 93 (1971), 753-809.

[12] D. LuNA. Sur les orbites fermées des groupes algébriques réductifs, Invent. Math., 16 (1972), 1-5. 
[13] T. MATSUKI. Double coset decompositions of reductive Lie groups arising from two involutions, J. Algebra, 197 (1997), 49-91.

[14] D. Panyushev. On spherical nilpotent orbits and beyond, Ann. Inst. Fourier, 49 (1999), 1453-1476.

[15] D. PAnYusheV. On invariant theory of $\theta$-groups, J. Algebra, 283 (2005), 655-670.

[16] D. PANYUSHEV. Semi-direct products of Lie algebras and their invariants, Publ. R.I.M.S., 43, no. 4 (2007), 1199-1257.

[17] D. PAnyushev. On the coadjoint representation of $\mathbb{Z}_{2}$-contractions of reductive Lie algebras, Adv. Math., 213 (2007), 380-404.

[18] R.W. RICHARDSON. Orbits, invariants, and representations associated to involutions of reductive groups, Invent. Math., 66 (1982), no. 2, 287-312.

[19] R.W. RichARDSON. Normality of $G$-stable subvarieties of a semisimple Lie algebra, In: "Algebraic Groups", Lecture Notes Math., Berlin: Springer, 1271 (1987), 243-264.

[20] R.W. Richardson. Derivatives of invariant polynomials on a semisimple Lie algebra, in: "Miniconference on harmonic analysis and operator algebras" (Canberra, 1987), 228-241, Proc. Centre Math. Anal. Austral. Nat. Univ., 15, Austral. Nat. Univ., Canberra, 1987.

[21] M. Rosenlicht. On quotient varieties and the affine embedding of certain homogeneous spaces, Trans. Amer. Math. Soc. 101 (1961), 211-223.

[22] W. SMOKE. Commutativity of the invariant differential operators on a symmetric space, Proc. Amer. Math. Soc., 19 (1968), 222-224.

[23] T.A. SPRINGER. Regular elements of finite reflection groups, Invent. Math. 25 (1974), 159-198.

[24] M. Vergne. Instantons et correspondance de Kostant-Sekiguchi, C. R. Acad. Sci. Paris Sér. I, t.320, no. 8 (1995), 901-906.

[25] E.B. VinBERG. Short $\mathrm{SO}_{3}$-structures on simple Lie algebras and associated quasielliptic planes. In: E. Vinberg (ed.), "Lie groups and invariant theory", AMS Translations, Series 2, 213 (2005), 243-270.

[26] Э.Б. Виньерг, А.Л. Онищик. "Семинар по группам Ли и алгебраическим группам". Москва: "Наука" 1988 (Russian). English translation: A.L. ONISHCHIK and E.B. VINBERG. "Lie groups and algebraic groups", Berlin: Springer, 1990.

[27] Э.Б. Виньерг, В.Л. Попов. "Теория инвариантов”, В кн.: Совр. пробл. матем., Фундам. направл., т. 55, с. 137-309. Москва: ВИНИТИ 1989 (Russian). English translation: V.L. Popov and E.B. VInBERG. "Invariant theory", In: Algebraic Geometry IV (Encyclopaedia Math. Sci., vol. 55, pp. 123-284) Berlin: Springer 1994.

[28] O.S. YAKIMOVA. Symmetric invariants of $\mathbb{Z}_{2}$-contractions, (in preparation).

INSTITUTE FOR INFORMATION TRANSMISSION PROBLEMS OF THE R.A.S.,

B. KARETNYI PER. 19, MOSCOW 127994, RUSSIA

E-mail address: panyushev@iitp.ru

URL: http://www.mccme.ru/ panyush 